\title{
DIRECT TRANSCRIPTION OF LOW-THRUST TRAJECTORIES WITH FINITE TRAJECTORY ELEMENTS
}

\section{Federico Zuiani}

PhD Candidate, School of Engineering, University of Glasgow, UK,

fzuiani@eng.gla.ac.uk

Massimiliano Vasile

Reader, Department of Mechanical Engineering, University of Strathclyde, UK

massimiliano.vasile@strath.ac.uk

Alessandro Palmas

PhD Candidate, School of Engineering, University of Glasgow, UK,

a.palmas.1@ research.gla.ac.uk

\section{Giulio Avanzini}

Assistant Professor, Department of Aerospace Engineering, Politecnico di Torino, Italy,

giulio.avanzini@polito.it

This paper presents a novel approach to the design of Low-Thrust trajectories, based on a first order approximated analytical solution of Gauss planetary equations. This analytical solution is shown to have a better accuracy than a second-order explicit numerical integrator and at a lower computational cost. Hence, it can be employed for the fast propagation of perturbed Keplerian motion when moderate accuracy is required. The analytical solution was integrated in a direct transcription method based on a decomposition of the trajectory into direct finite perturbative elements (DFPET). DFPET were applied to the solution of two-point boundary transfer problems. Furthermore the paper presents an example of the use of DFPET for the solution of a multiobjective trajectory optimisation problem in which both the total $\Delta V$ and transfer time are minimized with respect to departure and 
arrival dates. Two transfer problems were used as test cases: a direct transfer from Earth to Mars and a spiral from a low Earth orbit to the International Space Station.

\section{Nomenclature}

a

$\left[P_{1}, P_{2}, Q_{1}, Q_{2}\right]$

C

$e$

$h$

$i$

$I_{\mathrm{ij}}$

$J$

$L$

$p$

$r$

$t$

$\mathbf{u}$

ToF

X

$\alpha$

$\beta$

$\varepsilon$

$\Delta L$

$\Delta V$

$\mu$

$\omega$
$=$ semi-major axis, $\mathrm{km}$

$=$ Equinoctial Parameters

$=$ Constraints Vector

$=$ Eccentricity

$=$ Angular Momentum $\mathrm{km}^{2} / \mathrm{s}$

$=$ Inclination

$=$ Integral $_{\mathrm{ij}}$

$=$ Performance Index

$=$ True Longitude, $\mathrm{rad}$

$=$ Parameter or Semi-Latus Rectum, km

$=$ Radius, $\mathrm{km}$

$=$ Time, $\mathrm{s}$

$=$ Thrust acceleration, $\mathrm{km} / \mathrm{s}^{2}$

$=$ Time of Flight, $\mathrm{s}$

$=$ State vector

$=$ Thrust Azimuth Angle, rad

$=$ Thrust Elevation Angle, rad

$=$ Perturbation Parameter, $\mathrm{km} / \mathrm{s}^{2}$

$=$ True Longitude Arc Length, rad

$=$ Velocity Increment, $\mathrm{km} / \mathrm{s}$

$=$ Planetary Gravity Constant, $\mathrm{km}^{3} / \mathrm{s}^{2}$

$=$ Argument of Periapsis, $\mathrm{rad}$ 
$\Omega$

$\theta$

Subscripts

1

f
$=$ Right Ascension of the Ascending Node, rad

$=$ True Anomaly, rad

$=$ zero order term or initial value

$=$ first order term

$=$ final value

$=\mathrm{i}$-th element

\section{INTRODUCTION}

The design of low-thrust (LT) trajectories requires the definition of the thrust profile that satisfies a two-point boundary value problem. The scope of this work is to provide a computationally efficient way to determine a good approximated solution to this problem with a representation of the control profile comparable to more accurate but computationally expensive approaches.

In the literature, the problem has been tackled in a number of different ways ${ }^{1,2}$, generally classified in two families: indirect methods and direct methods. Indirect methods ${ }^{3,4,5}$ translate the design of a low-thrust trajectory into the solution of an optimal control problem and derive explicitly the associated first order optimality conditions. The first order optimality conditions are a system of mixed differential-algebraic equations (DAE). Shooting, multipleshooting, collocation and approximated analytical approaches have been proposed to solve the DAE system and satisfy the boundary conditions.

Direct methods ${ }^{6,7}$, instead, do not derive the optimality conditions but transcribe the differential dynamic equations of motion into a system of algebraic equations and then solve a nonlinear programming problem. Numerical integration and collocation techniques have been proposed to transcribe the differential dynamic equations.

Direct methods are generally computationally intensive while indirect methods can display some convergence problems. Both require some form of first guess solution. In the past decade, some low-fidelity approximation techniques have been proposed to generate the first guess solution ${ }^{8,9,10,11}$. The requirement for these low fidelity solutions is to be fast, because they are to be used to evaluate several thousands of possible trajectories, but they need 
to be accurate enough to provide a good estimation of the cost of the transfer (i.e. maximum thrust, $\Delta V$, total impulse, time of transfer, etc.). Thus, the use of low-fidelity solutions is not always straightforward. Sims and Flanagan ${ }^{12}$ first proposed a fast direct method based on the transcription of a low-thrust trajectory into a multi-burn transfer to generate a medium-fidelity solution at a low computational cost. This has also been used as a basis for global optimisation tools ${ }^{14,15}$. Recently, Sukhanov et. al. ${ }^{16}$ proposed a method in which multi-revolution LT trajectories are divided into sub-arcs and on each of them a linearised optimal control problem is solved.

In this paper, a direct method is presented where the trajectory is decomposed into a number of finite elements. Gauss planetary equations are solved over each element by means of a perturbative approach, for constant thrust modulus and direction. The trajectory is assumed to be an $\varepsilon$-variation of a Keplerian arc, where $\varepsilon$ is a 'small' acceleration term due to the low-thrust. A fast transcription of the trajectory into a nonlinear programming problem is thus obtained, the accuracy of which is controlled by the number of elements, assuming that every trajectory element remains a first order epsilon-variation of a Keplerian arc.

It will be shown how this approach can be used for the solution of computationally demanding multiobjective optimisation problems where both the mass of propellant and the transfer time need to be minimized.

\section{MODEL AND PROBLEM DEFINITION}

The perturbed two-body motion is expressed in terms of non-singular, equinoctial elements ${ }^{17}$. This is a particular parameterization that is not affected by singularities for orbits with zero-inclination (undefined line of nodes) or zero-eccentricity (undefined periapsis). The non-singular equinoctial elements are defined as follows:

$$
\mathbf{X}=\left\{\begin{array}{c}
a \\
P_{1}=e \sin (\Omega+\omega) \\
P_{2}=e \cos (\Omega+\omega) \\
Q_{1}=\tan \frac{i}{2} \sin \Omega \\
Q_{2}=\tan \frac{i}{2} \cos \Omega \\
L=\Omega+\omega+\theta
\end{array}\right\}
$$

The corresponding Gauss' planetary equations are: 


$$
\begin{gathered}
\frac{d a}{d t}=\frac{2 a^{2}}{h}\left[\left(P_{2} \sin L-P_{1} \cos L\right) \varepsilon \cos \beta \cos \alpha+\frac{p}{r} \varepsilon \cos \beta \sin \alpha\right] \\
\frac{d P_{1}}{d t}=\frac{r}{h}\left\{-\frac{p}{r} \cos L \cdot \varepsilon \cos \beta \cos \alpha+\left[P_{1}+\left(1+\frac{p}{r}\right) \sin L\right] \varepsilon \cos \beta \sin \alpha-P_{2}\left(Q_{1} \cos L-Q_{2} \sin L\right) \varepsilon \sin \beta\right\} \\
\frac{d P_{2}}{d t}=\frac{r}{h}\left\{-\frac{p}{r} \cos L \cdot \varepsilon \cos \beta \cos \alpha+\left[P_{2}+\left(1+\frac{p}{r}\right) \sin L\right] \varepsilon \cos \beta \sin \alpha-P_{1}\left(Q_{1} \cos L-Q_{2} \sin L\right) \varepsilon \sin \beta\right\} \\
\frac{d Q_{1}}{d t}=\frac{r}{2 h}\left(1+Q_{1}^{2}+Q_{2}^{2}\right) \sin L \cdot \varepsilon \sin \beta \\
\frac{d Q_{2}}{d t}=\frac{r}{2 h}\left(1+Q_{1}^{2}+Q_{2}^{2}\right) \cos L \cdot \varepsilon \sin \beta \\
\frac{d L}{d t}=\sqrt{\frac{\mu}{a^{3}}}-\frac{r}{h}\left(Q_{1} \cos L-Q_{2} \sin L\right) \varepsilon \sin \beta
\end{gathered}
$$

where $\varepsilon, \alpha$ and $\beta$ are respectively the modulus, azimuth and elevation of the perturbing acceleration in a radialtransversal reference frame as in Fig. 1 , where $O$ is the centre of the principal attracting body, $\hat{i}, \hat{j}, \hat{k}$ are the unit vectors of the inertial reference frame centred in $\mathrm{O}, \mathbf{r}$ is the position vector and $\hat{t}, \hat{r}, \hat{h}$ are the unit vectors transversal, radial and out of plane. $\mathbf{u}$ is the perturbative acceleration vector, which in the radial-transversal reference frame is defined as $\mathbf{u}=\varepsilon\left[\begin{array}{lll}\cos \alpha \cos \beta & \sin \alpha \cos \beta & \sin \beta\end{array}\right]$

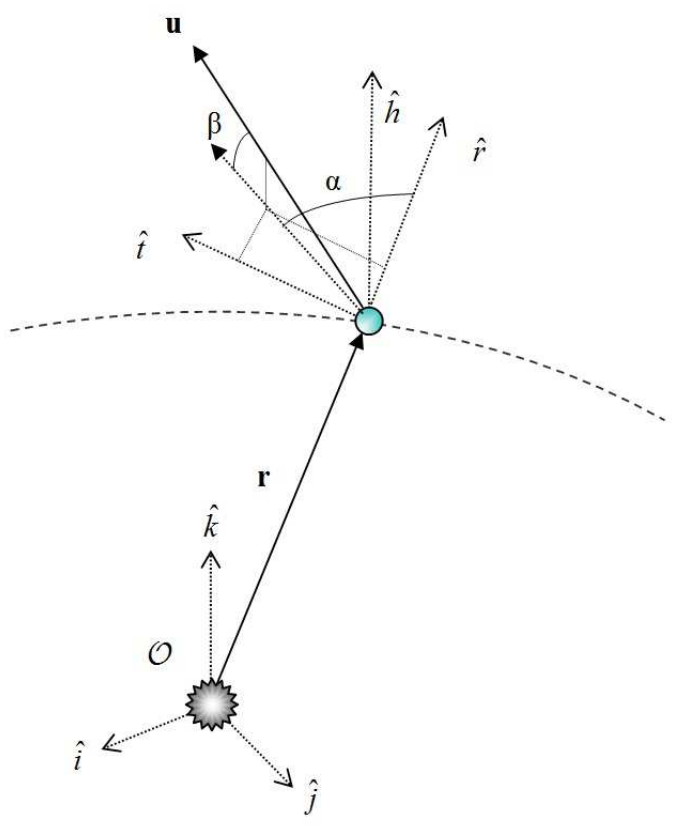

Fig. 1: Radial-transversal reference frame. 
If one takes $\varepsilon$ as the acceleration modulus delivered by a controllable engine along the directions defined by the angles $\alpha$ and $\beta$, the problem is to find the control law that satisfies the boundary conditions

$$
\left\{\begin{array}{l}
\mathbf{X}\left(t_{0}\right)=\overline{\mathbf{X}}_{0} \\
\mathbf{X}\left(t_{f}\right)=\overline{\mathbf{X}}_{f}
\end{array}\right.
$$

with $t_{f}=t_{0}+T o F$ and $T o F$ the time of flight, while minimizing the total $\Delta V$ of the transfer:

$$
\Delta V=\int_{t_{0}}^{t_{f}} \varepsilon(t) d t
$$

In mathematical terms the problem can be formulated as a typical Two-Point Boundary Value Problem (TPBVP) as follows:

$$
\begin{aligned}
& \min _{\varepsilon, \alpha, \beta} J=\int_{t_{0}}^{t_{f}} \varepsilon(t) d t \\
& \text { s.t. } \\
& \text { Eqs.[2] }
\end{aligned}
$$

\section{THE PERTURBATIVE APPROACH}

The system of nonlinear ordinary differential equations (ODE's) in [2] can be easily integrated with a generic numerical approach to obtain a very accurate solution. On the other hand, the solution of problem [5][5], requires multiple integrations of system [2]. Additionally, in large scale trajectory optimisation problems, Eqs. [5] might need to be solved thousands of times, making the use of numerical integrators even less appealing. For example, as it will be shown in Sec.V.III, a multi-objective optimisation of a simple transfer Earth Mars transfer requires the evaluation of some 40000 candidate solutions. Therefore it would be desirable to obtain a computationally cheaper, albeit less accurate, integration of [2]. Various authors have already proposed integrable forms for [2], either by introducing some simplifying assumptions ${ }^{18,19}$, or by reformulating the problem with a radically different approach ${ }^{20}$. Conversely, a perturbative approach, which employs low-thrust non-dimensional acceleration as the perturbation parameter, can be used to obtain a first-order analytical approximation of the evolution of the orbital parameters under the action of a perturbing force, which is constant in the $\hat{t}, \hat{r}, \hat{h}$ reference frame. 
Prior to the development of the first order approximation, Gauss's variational equations [2] are rewritten using the true longitude $L$ as the independent variable instead of time, by means of the derivation chain rule:

$$
\frac{d x}{d L}=\frac{d x}{d t} \frac{d t}{d L}
$$

where, the variable $x$ represents any of the first five equinoctial orbital elements in [1], while $d t / d L$ is given by

$$
\frac{d t}{d L}=\frac{r^{2}}{h}=\frac{h^{3}}{\left(1+P_{1} \sin L+P_{2} \cos L\right)^{2}}
$$

This latter expression also provides the additional equation needed to compute the time as a function of $L$. The orbital elements and time can be expanded up to first order in the perturbing parameter $\varepsilon$,

$$
\begin{aligned}
& a=a_{0}+\varepsilon a_{1} \\
& P_{1}=P_{10}+\varepsilon P_{11} \\
& P_{2}=P_{20}+\varepsilon P_{21} \\
& Q_{1}=Q_{10}+\varepsilon Q_{11} \\
& Q_{2}=Q_{20}+\varepsilon Q_{21} \\
& t=t_{00}+\varepsilon t_{11}
\end{aligned}
$$

where the zero-order terms, obtained for $\varepsilon=0$ correspond to the unperturbed Keplerian motion. The remaining terms, are computed by means of a technique based on standard perturbation theory ${ }^{23}$, applied to the set of first order ODEs [2]. This leads to a set of equations in the form:

$$
\begin{aligned}
& a=a_{0}+\varepsilon 2\left(\frac{h_{0} a_{0}}{\mu}\right)^{2} \cos \beta\left(\cos \alpha\left(P_{20} I_{s 2}-P_{10} I_{c 2}\right)+\sin \alpha I_{11}\right) \\
& P_{1}=P_{10}+\varepsilon \frac{h_{0}{ }^{4}}{\mu^{3}}\left(\cos \beta\left(-\cos \alpha I_{c 2}+\sin \alpha\left(P_{10} I_{13}+I_{s 2}+I_{s 3}\right)\right)+\sin \beta P_{20}\left(-Q_{10} I_{c 3}+Q_{20} I_{s 3}\right)\right) \\
& P_{2}=P_{20}+\varepsilon \frac{h_{0}{ }^{4}}{\mu^{3}}\left(\cos \beta\left(\cos \alpha I_{s 2}+\sin \alpha\left(P_{20} I_{13}+I_{c 2}+I_{c 3}\right)\right)+\sin \beta P_{10}\left(Q_{10} I_{c 3}-Q_{20} I_{s 3}\right)\right) \\
& Q_{1}=Q_{10}+\varepsilon 2 \frac{h_{0}{ }^{4}}{\mu^{3}} \sin \beta I_{s 3}\left(1+Q_{10}{ }^{2}+Q_{20}{ }^{2}\right) \\
& Q_{2}=Q_{20}+\varepsilon 2 \frac{h_{0}{ }^{4}}{\mu^{3}} \sin \beta I_{c 3}\left(1+Q_{10}{ }^{2}+Q_{20}{ }^{2}\right) \\
& t=t_{0}+h_{0}{ }^{3} I_{12}+\varepsilon \frac{\sqrt{a_{0}\left(1-P_{10}{ }^{2}-P_{20}{ }^{2}\right)}}{2} \\
& \left(\begin{array}{l}
\left.I_{c 3}\left(2 a_{0}\left(-3 P_{10} P_{20} P_{1}+P_{2}\left(2 P_{10}{ }^{2}-P_{20}{ }^{2}-2\right)\right)-3 a_{1} P_{20}\left(P_{10}{ }^{2}+P_{20}{ }^{2}-1\right)\right)+I_{s 3}\left(2 a_{0}\left(-3 P_{10} P_{20} P_{2}+P_{1}\left(2 P_{20}{ }^{2}-P_{10}{ }^{2}-2\right)\right)-3 a_{1} P_{10}\left(P_{10}{ }^{2}+P_{20}{ }^{2}-1\right)\right)\right) \\
-3 I_{13}\left(2 a_{0}\left(P_{10} P_{1}+P_{20} P_{2}\right)+a_{1}\left(P_{10}{ }^{2}+P_{20}{ }^{2}-1\right)\right)
\end{array}\right)
\end{aligned}
$$

where $I_{11}, I_{12}, I_{13}, I_{c 2}, I_{c 3}, I_{s 2}, I_{s 3}$, are the following integrals: 


$$
\begin{aligned}
& I_{11}\left(L_{F}\right)=\int_{L_{0}}^{L_{F}} \frac{1}{1+P_{10} \sin L+P_{20} \cos L} d L \\
& I_{12}\left(L_{F}\right)=\int_{L_{0}}^{L_{F}} \frac{1}{\left(1+P_{10} \sin L+P_{20} \cos L\right)^{2}} d L \\
& I_{13}\left(L_{F}\right)=\int_{L_{0}}^{L_{F}} \frac{1}{\left(1+P_{10} \sin L+P_{20} \cos L\right)^{3}} d L \\
& I_{c 2}\left(L_{F}\right)=\int_{L_{0}}^{L_{F}} \frac{\cos L}{\left(1+P_{10} \sin L+P_{20} \cos L\right)^{2}} d L \\
& I_{c 3}\left(L_{F}\right)=\int_{L_{0}}^{L_{F}} \frac{\cos L}{\left(1+P_{10} \sin L+P_{20} \cos L\right)^{3}} d L \\
& I_{s 2}\left(L_{F}\right)=\int_{L_{0}}^{L_{F}} \frac{\sin L}{\left(1+P_{10} \sin L+P_{20} \cos L\right)^{2}} d L \\
& I_{s 3}\left(L_{F}\right)=\int_{L_{0}}^{L_{F}} \frac{\sin L}{\left(1+P_{10} \sin L+P_{20} \cos L\right)^{3}} d L
\end{aligned}
$$

The integrals in Eqs. [10] are evaluated between the initial and final values of $L$. A closed-form solution is available in the complex domain for all the integrals necessary for the evaluation of the perturbative expansions of equinoctial orbit parameters. The derivation was carried out by means of a symbolic manipulation software and it is omitted here for the sake of conciseness. As for the time equation, twelve more integrals are needed in order to have the exact evaluation of the complete first order expansion, but unfortunately only four of them allow for an analytical solution.

The use of numerical quadrature was not considered here for two reasons: on one side it would have partially spoiled the elegance of a fully analytical approximate solution and, at the same time, a mixed analytical-numerical method would have been characterized by an increased computational burden. Provided that the analytical terms available for the expansion of time in perturbative terms improves the solution over the corresponding zero-order estimate, these terms are included in the determination of the time interval over each trajectory arc, although, in rigorous mathematical terms, the estimate of time is limited to a zero-order expansion with a correction. Nonetheless, the accuracy obtained for the present applications appears to be sufficient for the aims of the present work.

Once the analytical expressions for $a_{1}(L), P_{11}(L), P_{21}(L), Q_{11}(L)$, and $Q_{21}(L)$ are available, together with $t_{00}(L)$ and $t_{11}(L)$, the five variations of the orbital parameters and time are known as a first order approximated function of the true longitude $L$. Thus, one can analytically propagate the non-singular elements, either backward or forward in $L$, for an arbitrary set of initial (final) conditions and control force components, expressed in terms of magnitude and two angles. 
The length of the arc along which it is possible to propagate the evolution of $a_{1}, P_{11}, P_{21}, Q_{11}$, and $Q_{21}$, for a given set of equinoctial parameters initial values, depends on the required accuracy. Conversely, if the accuracy is assigned, the length of the arc is inversely proportional to the ratio between thrust and local gravitational acceleration. All these aspects will be quantitatively analyzed in Sec.IV.I.

\section{FINITE PERTURBATIVE ELEMENT TRANSCRIPTION}

The first-order approximation of the non-singular elements in [8] cannot be directly applied to the solution of problem [5] because the control acceleration has to be constant along the integrated trajectory arc. Furthermore, there is no independent control of the accuracy and of the arc length. In order to overcome these two issues the trajectory is decomposed into finite elements, each one representing an arc of prescribed amplitude. On each element, an approximated solution to Eqs. [2] is computed by means of the perturbative approach. All the elements are then linked together to form the complete trajectory. In analogy to Direct Finite Element Transcription ${ }^{25,26}$, this novel transcription approach is called Direct Finite Perturbative Element Transcription (DFPET) method. A similar transcription method can be found in the work of Sims and Flanagan ${ }^{12}$. The approach of Sims and Flanagan makes use of a zero-order approximation of the perturbed Keplerian motion by decomposing the trajectory into $n$ sub-arcs, with each sub-arc describing a leg of unperturbed Keplerian motion. The change in momentum due to a continuous thrust is lumped into $\Delta V$ discontinuities at the edges of each sub-arc. The main advantage resides in the fast closedform computation of each Keplerian arc and its variation with respect to the states at the beginning of the arc. By analogy, in DFPET the simple Keplerian model with discrete $\Delta V$ impulses is replaced by a first-order perturbed Keplerian model with constant thrust along each sub-arc (see Fig. 2). 


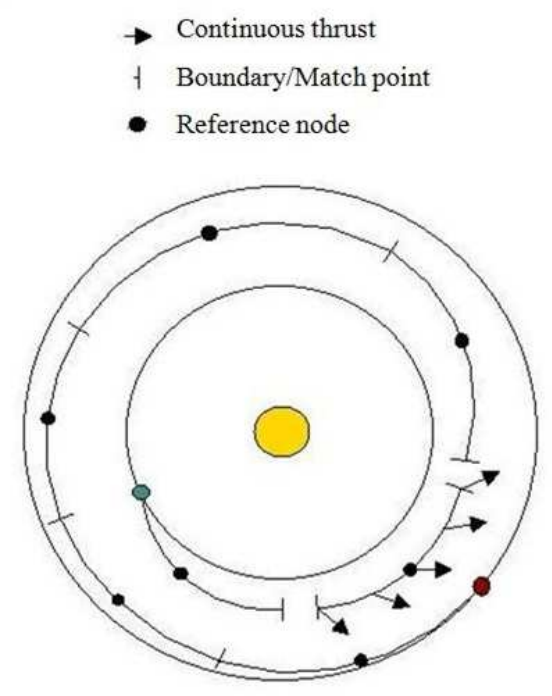

Fig. 2: LT Direct Finite Perturbative Element Transcription Method.

In DFPET, the $i$-th arc of amplitude $\Delta L_{i}$ is defined by the following quantities: $\mathbf{X}^{\mathbf{m}}$, the six Equinoctial parameters at the mid-point of the arc and the three control parameters $\varepsilon, \alpha$ and $\beta$. To obtain the boundary points of the element, the perturbed motion is analytically propagated backward and forward along a subarc with amplitude $\Delta L / 2$. The midpoint along the arc $\Delta L$ is chosen as the base-point for the analytical propagation to improve accuracy, since the error increases superlinearly with amplitude of the arc and is proportional to $\varepsilon$ (see Fig. 8). Thus, a dual-sided propagation in the form:

$$
\left\{\begin{array}{l}
\mathbf{X}^{+}=f\left(\mathbf{X}^{\mathbf{m}}, \frac{\Delta L}{2}, \varepsilon, \alpha, \beta\right) \\
\mathbf{X}^{-}=f\left(\mathbf{X}^{\mathbf{m}},-\frac{\Delta L}{2}, \varepsilon, \alpha, \beta\right)
\end{array}\right.
$$

provides a better accuracy than a single forward one over the arc-length of equivalent total length $\Delta L$. All arcs are then interconnected by imposing matching conditions at their boundaries (see Fig. 3). The proposed dual-sided propagation is different from what is usually done in other Multiple Shooting methods, in which the propagation is carried out only forwards. 


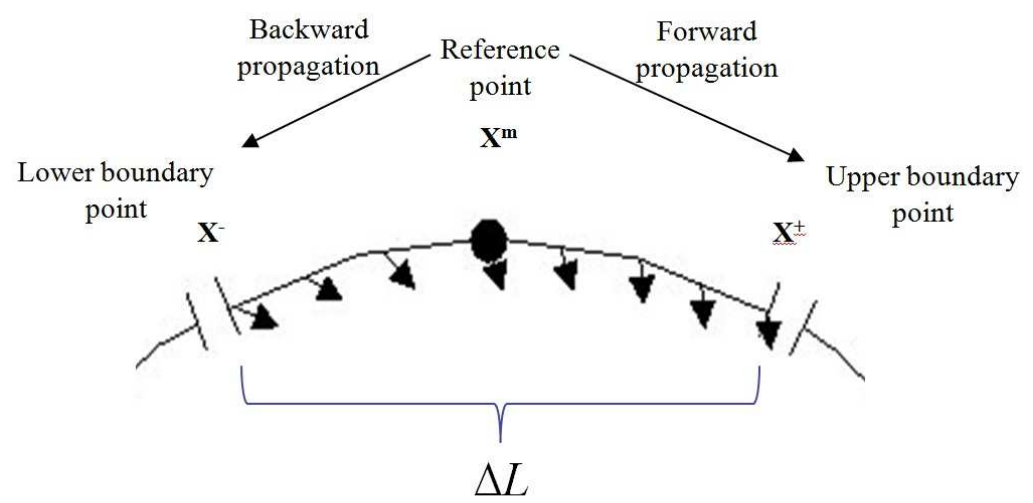

Fig. 3: DFPET with centered reference node.

\section{IV.I Accuracy Analysis}

In order to test the integration accuracy of the Perturbative Finite Element Transcription, the backward and forward integration over a single arc was run for a wide range of time intervals, a constant transversal acceleration of $2.5 \times 10^{-5} \mathrm{~m} / \mathrm{s}^{2}$ and an initially circular orbit at $1 \mathrm{AU}$ from the Sun. Time intervals ranged from 0.5 up to 100 days. Note that the centered reference node of the Perturbative Finite Element was adjusted in order to match, at the lower boundary, the above mentioned conditions of a $1 \mathrm{AU}$ heliocentric circular orbit. For each element size, the computed final state (i.e. the one at the upper boundary) was compared against the result of the integration of [2] with a simple implementation of the Modified Euler Method ${ }^{13}$, and the MatLab ${ }^{\circledR}$ function ode113 (that implements a variable-order Adams-Bashforth-Moulton Predictor-Corrector method). Other algorithms of the ode family (e.g. ode45) were also tested, and ode113 was chosen as being the fastest among them for a given accuracy requirement. The numerical integration was started from the lower boundary of the Perturbative Finite Element. The required relative and absolute accuracies were both set at $10^{-13}$, in order to have a very accurate solution to which to compare the results of the propagation with DFPET and the Modified Euler Method. Thus, the results of ode113 were used as a reference to compute the relative error on the final state as shown in Fig. 5 and Fig. 6. Moreover, CPU time for the case in which ode113 is allowed to use relaxed tolerances (both set at $10^{-3}$ ) is also included in Fig. 5 for a further comparison. 


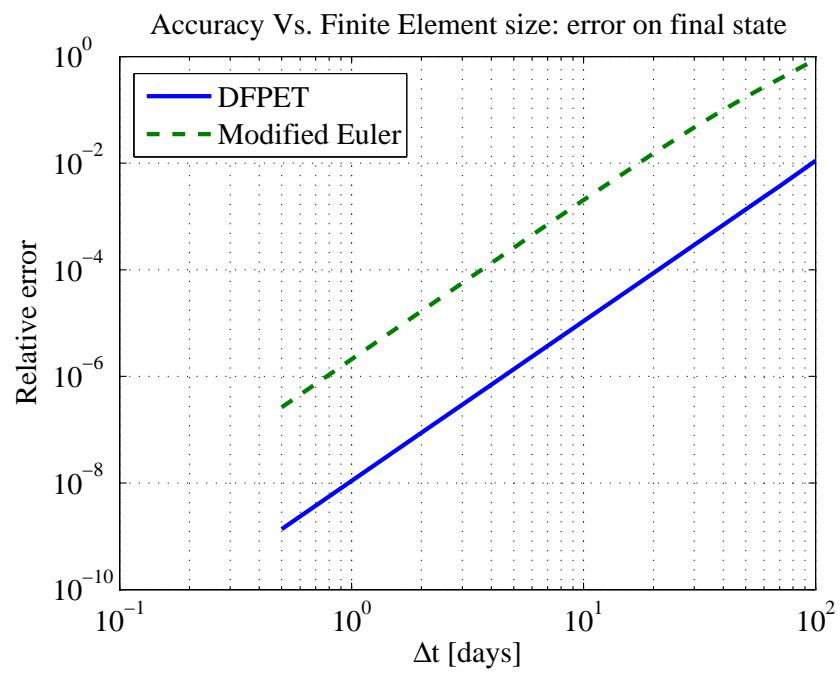

Fig. 4: Error on final state w.r.t. DFPET size for a heliocentric orbit.

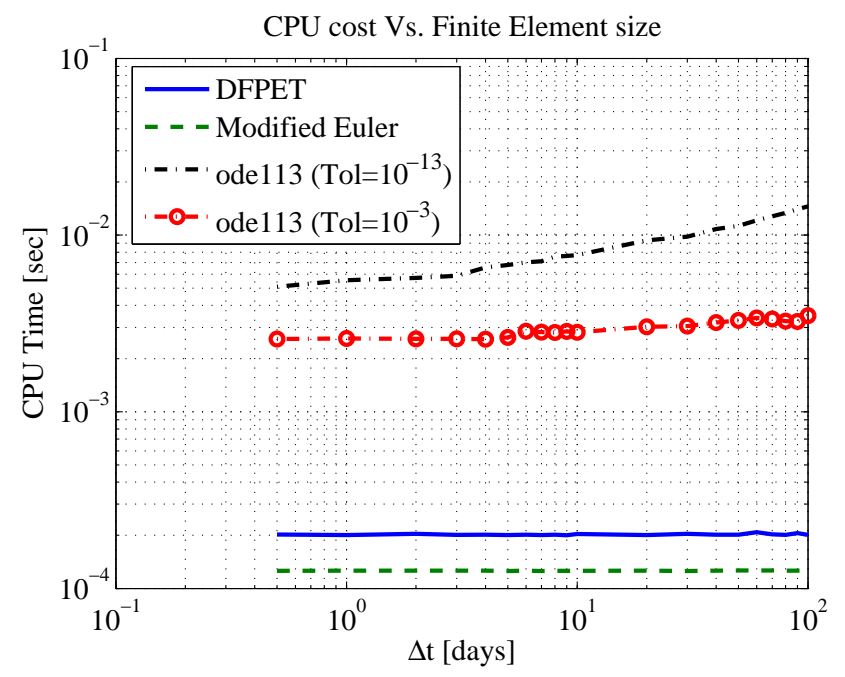

Fig. 5: CPU cost w.r.t. DFPET size for a heliocentric orbit.

In these tests, the DFPET transcription displays a second-order behaviour with respect to temporal size of the element and a good accuracy even with a relatively large size of the element (see Fig. 4). It should be noted that, in this test, the perturbation force is equivalent to a thrust of $0.5 \mathrm{~N}$ continuously acting on a $2000 \mathrm{~kg}$ spacecraft. This means that the ratio between the perturbative acceleration and the local gravity is relatively high. Finally, the DFPET method has a computational cost only marginally higher than the Modified Euler method (see Fig. 5) but still at least one order of magnitude lower than the numerical integration with ode113, even with relaxed tolerances. 
A similar test was carried out also by propagating a perturbed LEO orbit for an element size ranging from 0.5 to 5 days, roughly equivalent to 50 revolutions.

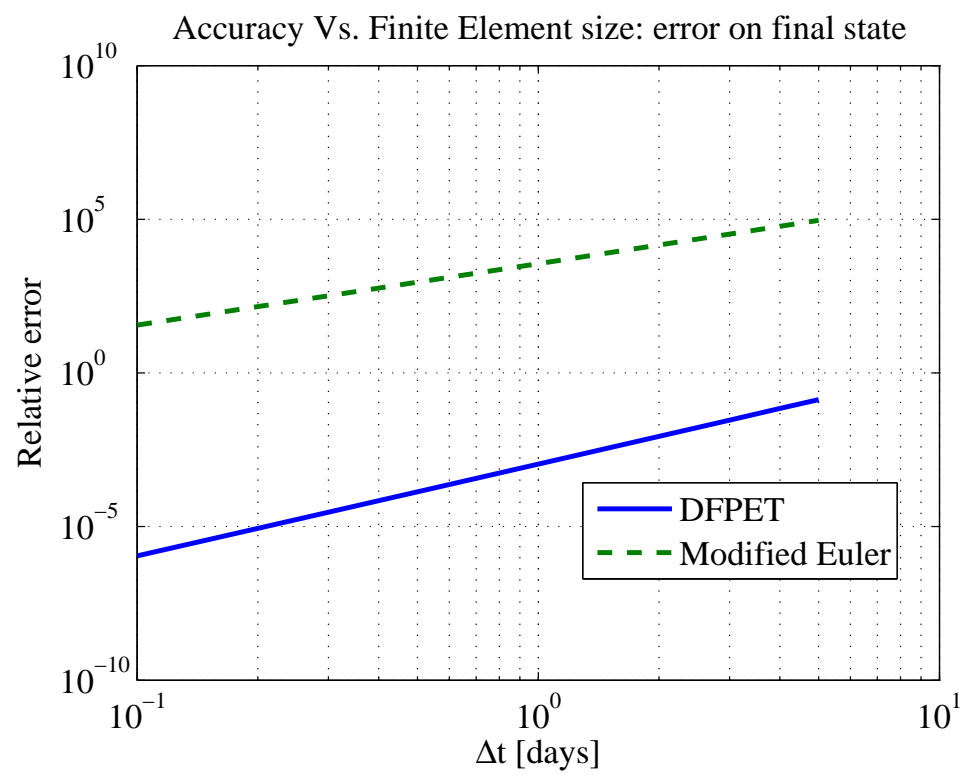

Fig. 6: Error on final state w.r.t. DFPET size for LEO propagation.

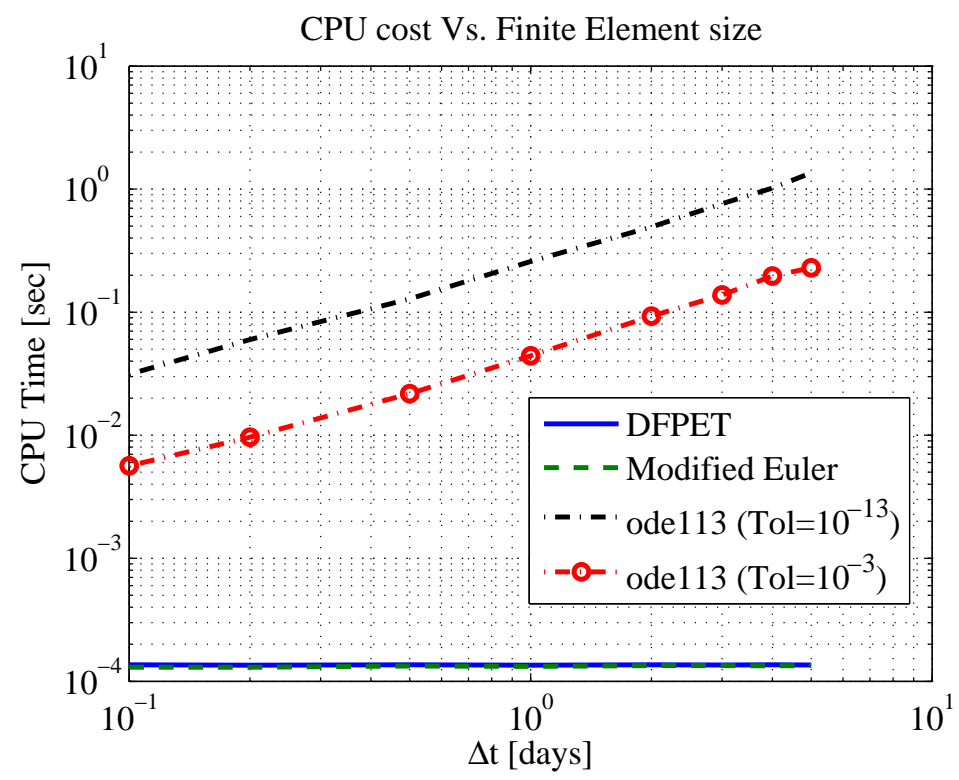

Fig. 7: CPU cost w.r.t. DFPET size for LEO propagation.

Here the advantage of the analytic propagation is even more evident, as it outperforms the Modified Euler Method (see Fig. 6), and is at least 60 times faster than the numerical integration with ode113 (see Fig. 7). This is 
easily explained by the fact that in LEO the gravitational force of the Earth is many times higher than the perturbation force. It should also be noted that the analytic propagation is able to provide an accurate estimate even with only one (or even a fraction of) Finite Element per revolution.

Finally, a simple test was performed to evaluate the accuracy with respect to the ratio between the thrust acceleration and the local gravitational force. An initial circular low Earth orbit was propagated analytically for 0.5 days with various levels of transverse acceleration and the results were again compared against the numerical integration of the same orbit arc with ode113. The relative error in the final state is shown in Fig. 8.

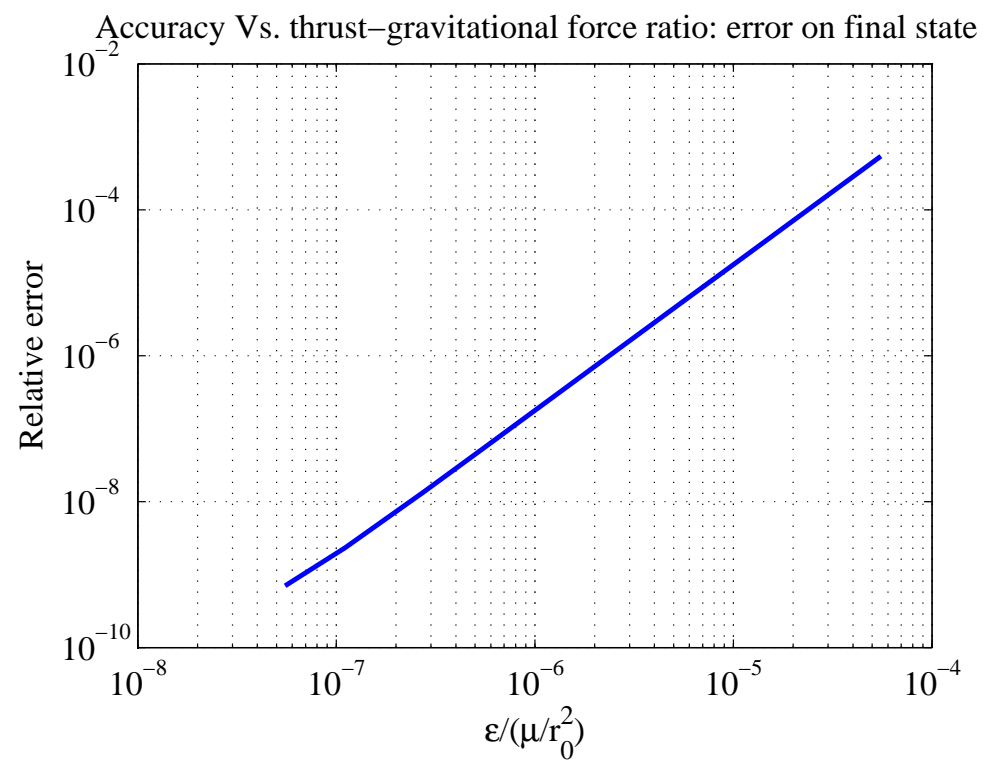

Fig. 8: Error on final state w.r.t. thrust-gravitational force ratio.

\section{IV.II Solution of the TPBVP Orbit Transfer Problem}

The DFPET approach can now be applied to the solution of problem [5], leading to the following system of nonlinear algebraic equations: 


$$
\begin{gathered}
\min _{\mathbf{u}} J=\sum_{i=1}^{n_{F P E T}} \varepsilon_{i} \Delta t_{i} \\
\text { s.t. } \mathbf{C}_{\mathbf{e q}}=\left\{\begin{array}{c}
\mathbf{X}_{1}^{-}-\mathbf{X}_{0} \\
\mathbf{X}_{i}^{+}-\mathbf{X}_{i+1}^{-}, i=2, \ldots, n_{F P E T}-1 \\
\mathbf{X}_{n_{F P E T}}^{+}-\mathbf{X}_{f} \\
\frac{T o F}{T o}-T o F=\sum_{i=1}^{n_{F P E T}} \Delta t_{i}
\end{array}\right\}=0 \\
\varepsilon_{i} \leq \varepsilon_{\text {max }}, i=1, \ldots, n_{F P E T}
\end{gathered}
$$

The decision variables are the control vector components and the first five equinoctial elements of the midpoint of each arc. The vector $\mathbf{u}$ in Eqs.[12] collects all the values of the control vector components for all the arcs. Equality constraints are given by the matching condition between adjacent sub-arcs. The first and last sets of rows in $\mathbf{C}_{e q}$ contain respectively the boundary conditions on the initial and final state and the last row contains the constraint on the time of flight: the required time of flight $\overline{T o F}$ has to match the one computed from the sum of the times of flight of the single sub-arcs as $T o F=\sum_{i=1}^{n_{F P E T}} \Delta t_{i}=\sum_{i=1}^{n_{F P E T}}\left(t_{i}-t_{i-1}\right)$. Note that continuity conditions with respect to the longitude are automatically satisfied since all the analytical expressions for the variation of the equinoctial elements are already parameterised with respect to $L$. Therefore, the matching constraints apply only to the remaining five equinoctial elements. Furthermore, the total longitude $\Delta L_{t o t}$ covered by the trajectory arc is easily determined as $\Delta L_{\text {tot }}=L_{f}-L_{0}=\sum_{i=0}^{n_{F P E T}} \Delta L_{i}$. It should also be noted that, to increase the number of complete revolutions of the trajectory, it is sufficient to increase $L_{f}$, and thus $\Delta L_{t o t}$, by multiples of $2 \pi$. In the current implementation only a uniform mesh (with respect to $L$ ) was considered.

Limits on the maximum delivered thrust are introduced as limits on the maximum perturbative acceleration. This is not entirely correct since, in fact, while the maximum thrust is constant, the maximum acceleration available gradually increases with time due to a gradual decrease of spacecraft mass. However, for the sake of the calculations in this paper, this approach is acceptable and allows for directly enforcing a reasonable upper limit on a decision variable. 
Problem [12] was solved by means of the MatLab ${ }^{\circledR}$ function fmincon, implementing a Sequential Quadratic Programming (SQP) method. Given $n_{F P E T}$ sub-arcs, the problem has $8 n_{F P E T}$ decision variables and $5\left(n_{F P E T}+1\right)+1$ scalar equality constraints. Because each control element is decoupled from the others, the Jacobian matrix is highly sparse with the structure shown in Fig. 9. The figure shows an example with 10 sub-arcs, i.e. with 80 variables and 56 scalar constraints. Size of the full matrix is thus 4480 but it has only 800 non-zero elements.

The sparsity pattern could be divided in three main regions: the left diagonal band, the right diagonal band and the lowermost row. The first corresponds to the derivatives of the constraints between adjacent sub arcs (as in Eqs.[12]) w.r.t. the modulus, azimuth and elevation of the thrust acceleration of each sub-arc. The band is composed by five-by-three sub-matrices each of which is basically the Jacobian of the first five Equinoctial elements at the lower (or upper) boundary as a function of $\varepsilon, \alpha, \beta$. The two zero elements within each sub-matrix correspond to the derivatives of $Q_{1}$ and $Q_{2}$ w.r.t. $\alpha$ which are always null as can be easily seen from Eqs.[9].

The second region corresponds to the derivatives of the matching constraints with respect to the reference nodes. Here again one can see a band structure with five-by-five sub-matrices. The two zero elements are in this case the derivatives of the semi-major axis w.r.t. $Q_{1}$ and $Q_{2}$.

Finally, the lowermost row is composed of the derivatives of the time of flight w.r.t. to all the variables.

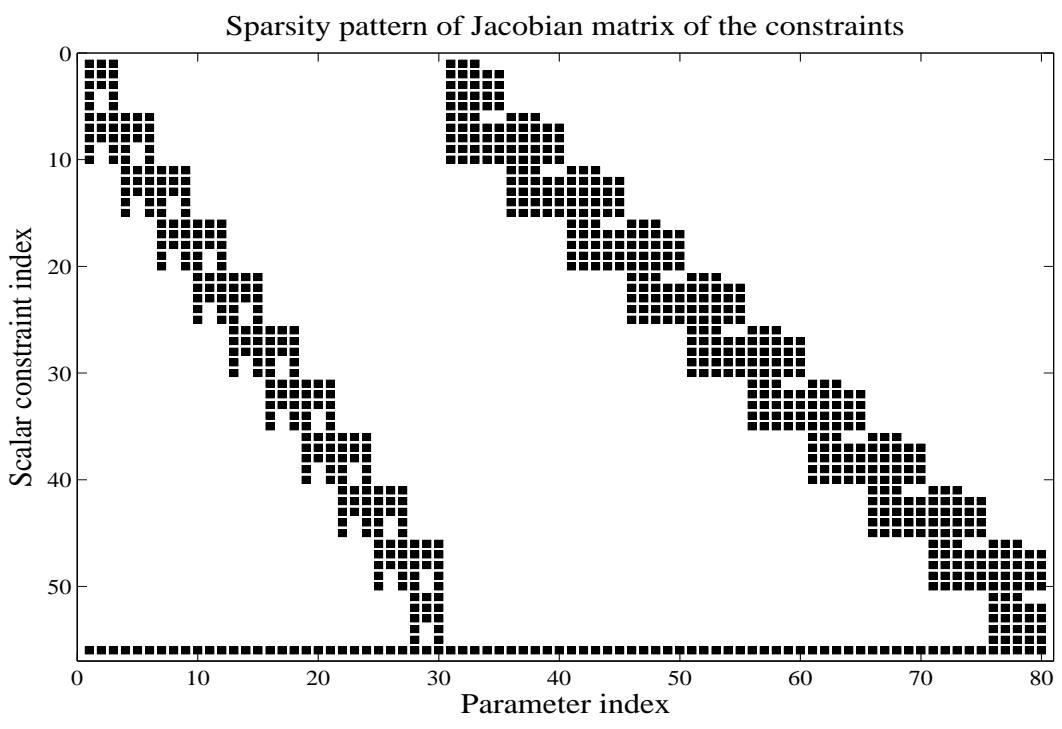

Fig. 9: Sparsity pattern of the Jacobian of the constraint function. 


\section{CASE STUDIES}

To test the proposed transcription method on realistic transfer problems, two cases were considered: the first is a direct, rendezvous transfer from Earth to Mars while the second is an orbit rising from a $260 \mathrm{~km}$ circular Low Earth Orbit (LEO) to the International Space Station (ISS) orbit. Both problems were solved first as a simple boundary problem and subsequently as a Multi-Objective problem using Epic, a population based Memetic algorithm developed by the authors and already described in previous works ${ }^{28}$.

\section{V.I Low-Thrust Earth-Mars Spiral}

For the simple boundary problem solution, the objective is that of finding a $\Delta V$ optimal transfer between Earth and Mars departing from Earth at $t_{0}=5600$ MJD2000, with a time of flight of 1095 days, and with 2 complete revolutions. Maximum acceleration was set at $2.5 \times 10^{-8} \mathrm{~km} / \mathrm{s}^{2}$, equivalent to a thrust of $0.5 \mathrm{~N}$ applied to a $2000 \mathrm{~kg}$ spacecraft. Initial guess was given by a constant, transversal thrust profile of magnitude half the maximum acceleration. The orbit was modelled with 20 Finite Elements. The problem was solved with the fmincon active-set algorithm. setting a tolerance of $10^{-8}$ both on constraint satisfaction and optimality condition.

The solution obtained has a total $\Delta V$ of $5.6388 \mathrm{~km} / \mathrm{s}$. In order to check the accuracy of the analytical solution, the optimal thrust profile was numerically integrated forward in time to calculate the final state. The maximum relative error between analytical and numerical final state was $3 \times 10^{-3}$.

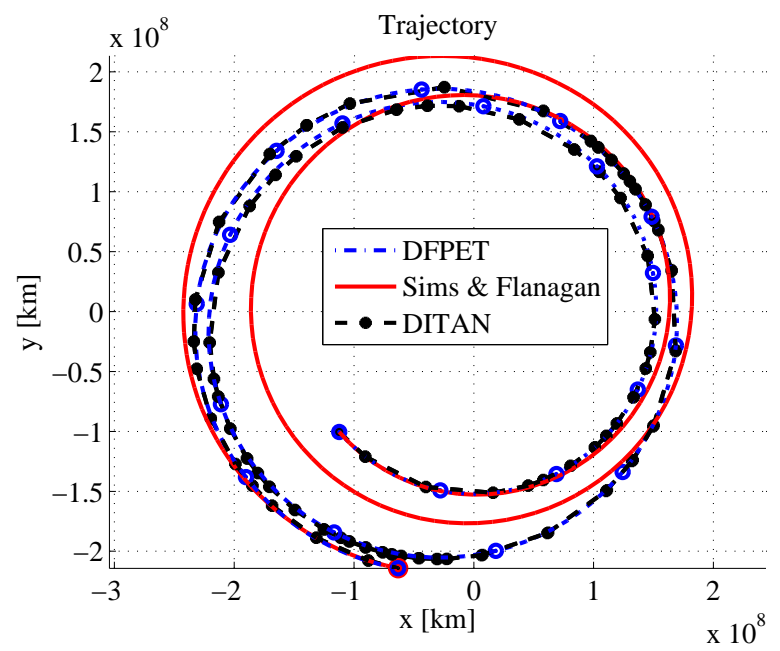

Fig. 10: Comparison of the optimized trajectories. 


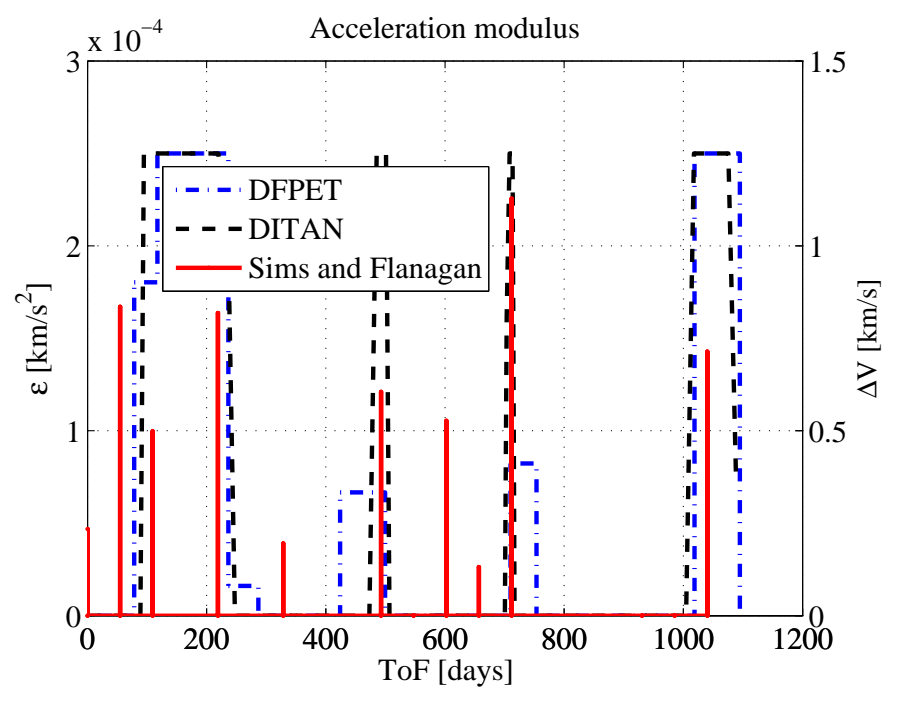

Fig. 11: Thrust modulus for Earth-Mars LT transfer.

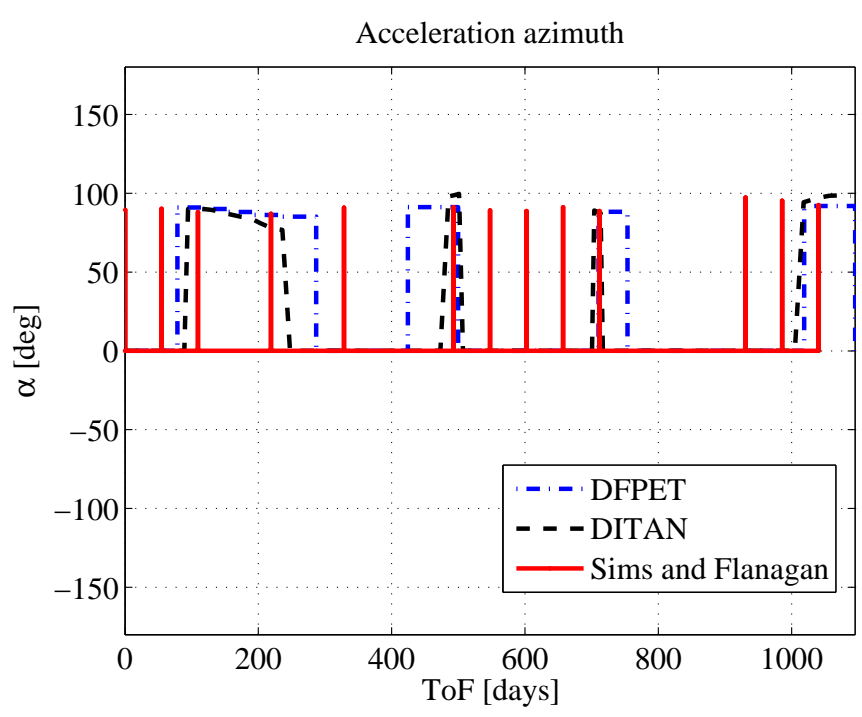

Fig. 12: Acceleration azimuth $\alpha$ for Earth-Mars LT transfer. 


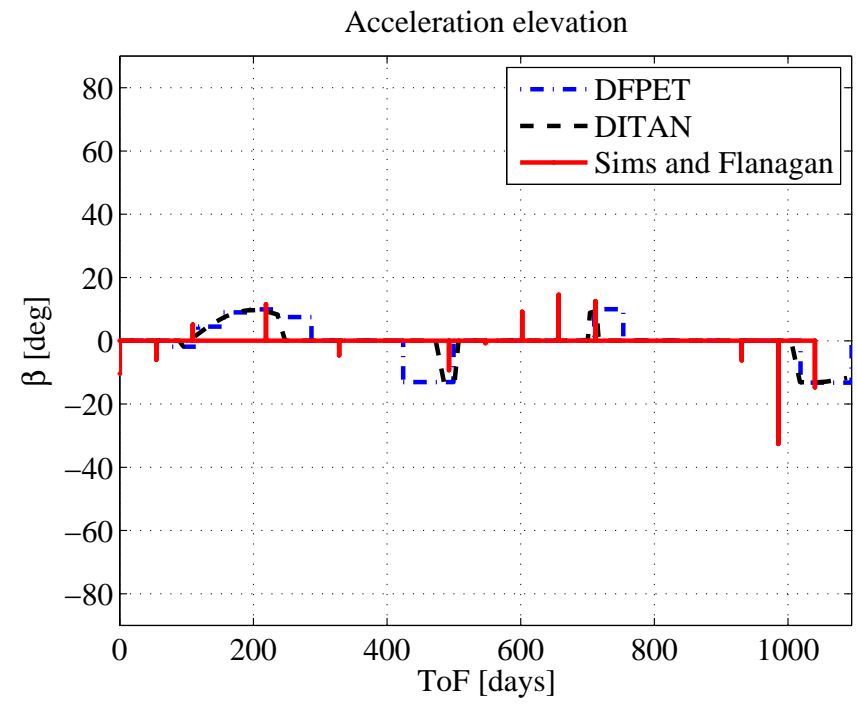

Fig. 13: Acceleration elevation $\beta$ for Earth-Mars LT transfer.

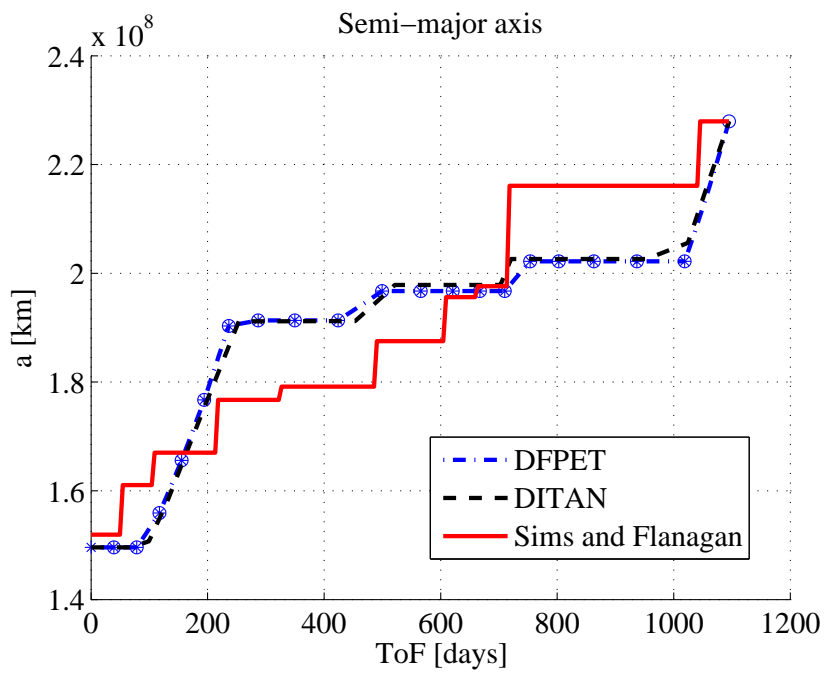

Fig. 14: Variation of Keplerian Elements for Earth-Mars LT transfer: $a$. 


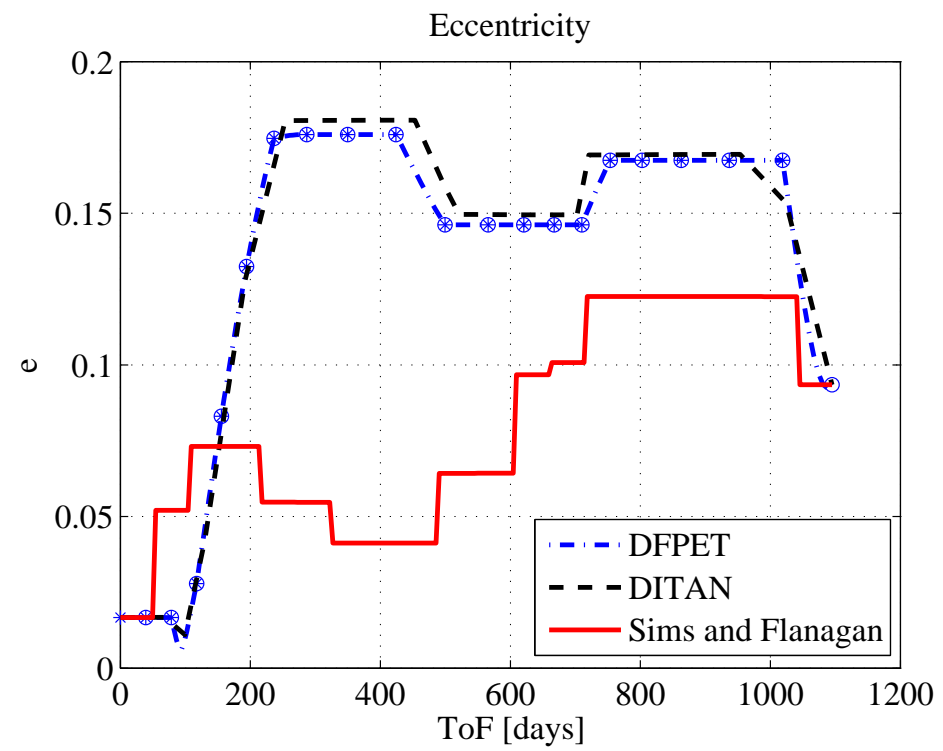

Fig. 15: Variation of Keplerian Elements for Earth-Mars LT transfer: $e$.

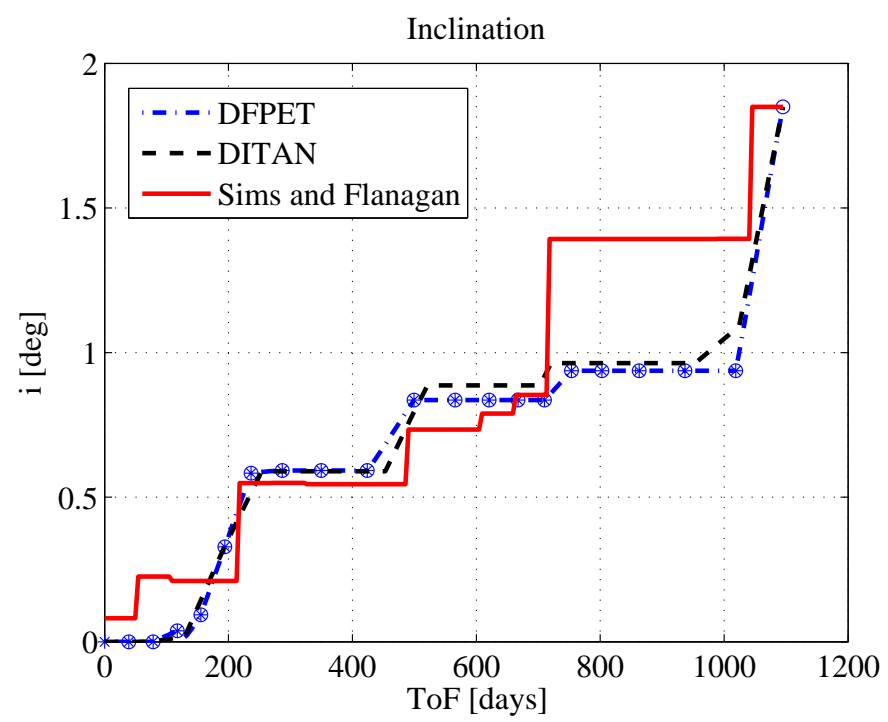

Fig. 16: Variation of Keplerian Elements for Earth-Mars LT transfer: $i$. 


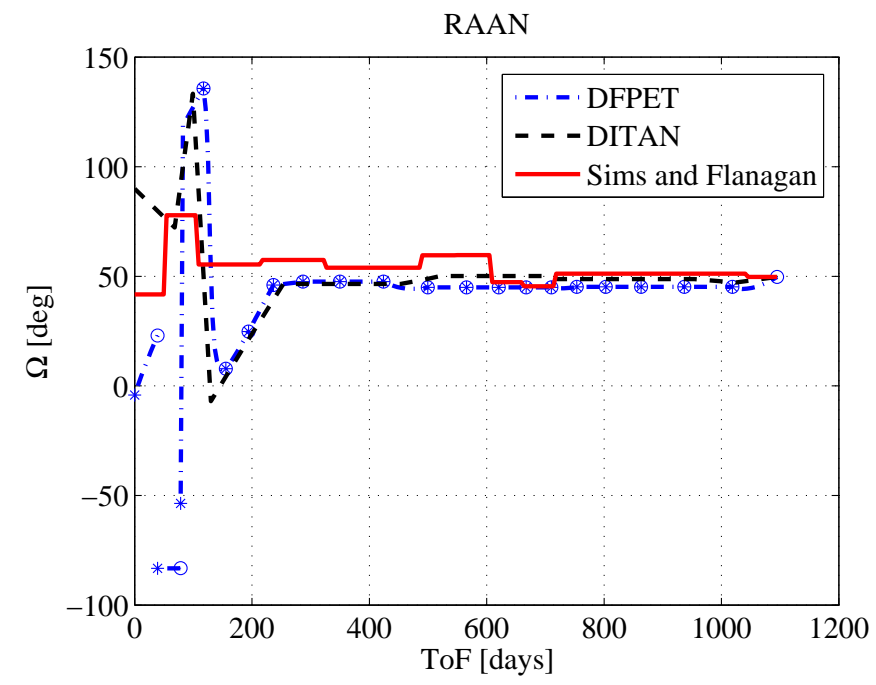

Fig. 17: Variation of Keplerian Elements for Earth-Mars LT transfer: $\Omega$.

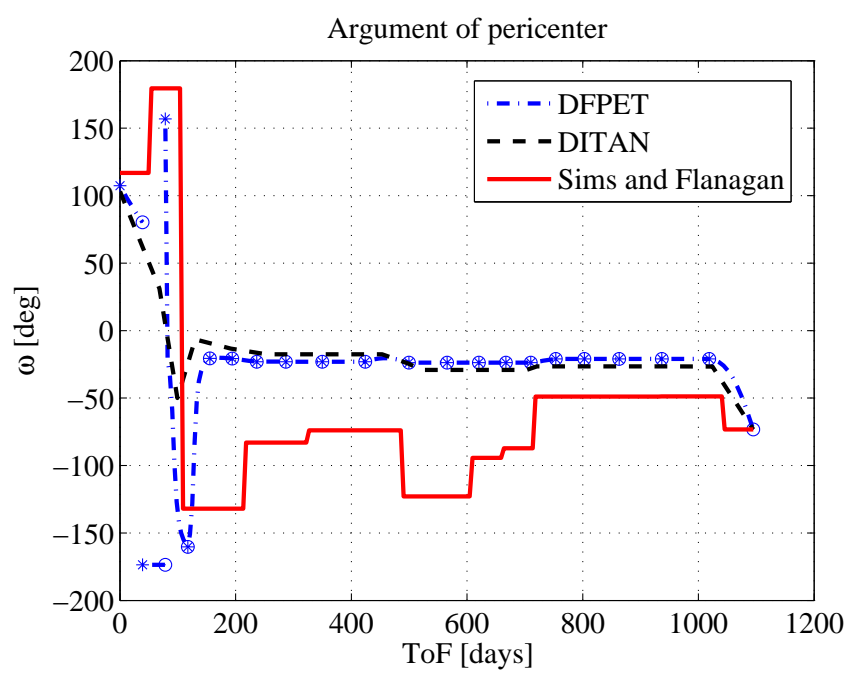

Fig. 18: Variation of Keplerian Elements for Earth-Mars LT transfer: $\omega$.

Fig. 11 shows the time history of the thrust modulus while Fig. 12 and Fig. 13 show its azimuth and elevation respectively. Note that, for ease of visualization of the thrusting or coasting arcs, the angles have been plotted as equal to zero when the corresponding thrust modulus is zero. The thrust profile displays a typical on-off structure with four thrusting arcs concentrated around the pericenter and apocenter. The azimuth angle (see Fig. 12) is almost constantly at $90^{\circ}$, which translates into a quasi-transverse in-plane component of the thrust. The small out-of-plane component (see Fig. 13) is due to the small change in inclination between departure and arrival orbit. Fig. 14 to Fig. 18 show the time history of the Keplerian elements during the transfer. The semi-major axis increases monotonically 
(see Fig. 14), with the largest variations along the first and last thrusting arcs. The inclination (see Fig. 16) shows also a similar pattern, while the eccentricity (see Fig. 15) remains constant at about 0.17 for most of the transfer and then decreases to about 0.1 to match the eccentricity of the arrival orbit.

Note that, the discontinuities in both the plots of $\Omega$ and $\omega$ (see Fig. 17 and Fig. 18 respectively) are due to the small initial inclination that causes numerical problems in the derivation of the Keplerian elements from the equinoctial non-singular elements. There is no mismatch in the value of the equinoctial elements instead

The same problem was solved with an implementation of the Sims and Flanagan method, and with DITAN, an optimal control solver based on a direct transcription with Finite Elements on spectral basis ${ }^{25,26}$. As for the DFPET solution, 20 sub-arcs were used to transcribe the transfer problem with Sims and Flanagan and the resulting nonlinear programming problem was solved with fmincon setting the tolerance for both constraint satisfaction and optimality condition to $10^{-8}$.

DITAN uses $\mathrm{SNOPT}^{27}$ as nonlinear programming solver. A first solution was computed with 12 finite elements and the result was then improved by increasing the number of elements to 19 . The maximum constraint violation was set to $10^{-8}$ and the required optimality to $10^{-6}$.

\begin{tabular}{lllll}
\hline & FPET & S\&F & DITAN & DITAN \\
& & & & (refined) \\
\hline Elements & 20 & 20 & 12 & 19 \\
$\Delta V[\mathrm{~km} / \mathrm{s}]$ & 5.6388 & 5.6859 & 5.6429 & 5.5401 \\
Iterations & 63 & 240 & 4602 & 11064 \\
$t_{C P U}[\mathrm{sec}]$ & 10.88 & 21.37 & 1045.3 & 3582.73 \\
\hline
\end{tabular}

Table 1: Performance comparisons for three different LT optimisation methods.

Table 2 summarises optimisation results for the three different methods and shows that both the DFPET and Sims and Flanagan give reliable first guess figures for the $\Delta V$ cost of the transfer. On the other hand, it also shows the inherent advantages of the DFPET method compared to Sims and Flanagan. While the total $\Delta V$ of the former is only marginally better, its required number of fmincon iterations, and thus CPU time, is much lower. Moreover, if one considers the trajectory shape (see Fig. 10) and thrust modulus (see Fig. 11) and angles (see Fig. 12 and Fig. 13), the 
DFPET solution compares well with the solution given by DITAN. In this sense, the FPET solution could be considered as a good sub-optimal solution of this transfer problem.

\section{V.II LEO-to-ISS Orbit Rising}

The second test case considers a hypothetic orbit transfer between the Ariane 5ATV injection orbit and the ISS orbit, requiring an altitude increase of $95 \mathrm{~km}$. The boundary problem is formulated in an analogous way as the EarthMars case, although the ISS motion is modelled as a simple, planar, Keplerian motion and the injection orbit is assumed to be coplanar to the latter. The parameters of the departure LEO are considered fixed, with the exception of the initial true anomaly $\vartheta_{0}$. Departure time is also considered to be fixed, but the Time of Flight determines the position of the rendezvous with the ISS. It is therefore essential to define the optimal phasing between the departure from LEO and the encounter with the ISS.

The problem is to optimize the $\Delta V$ for a transfer with $\vartheta_{0}=210^{\circ}, T o F=2.02$ days and $n_{r e v}=32$. Forty finite perturbative elements were used in the optimisation. The optimised trajectory has a $\Delta V$ of $54.9 \mathrm{~m} / \mathrm{s}$. The accuracy is $10^{-5}$, a truly remarkable result considering the high number of revolutions. It should also be noted, that convergence was also very fast, with fmincon requiring only three iterations.

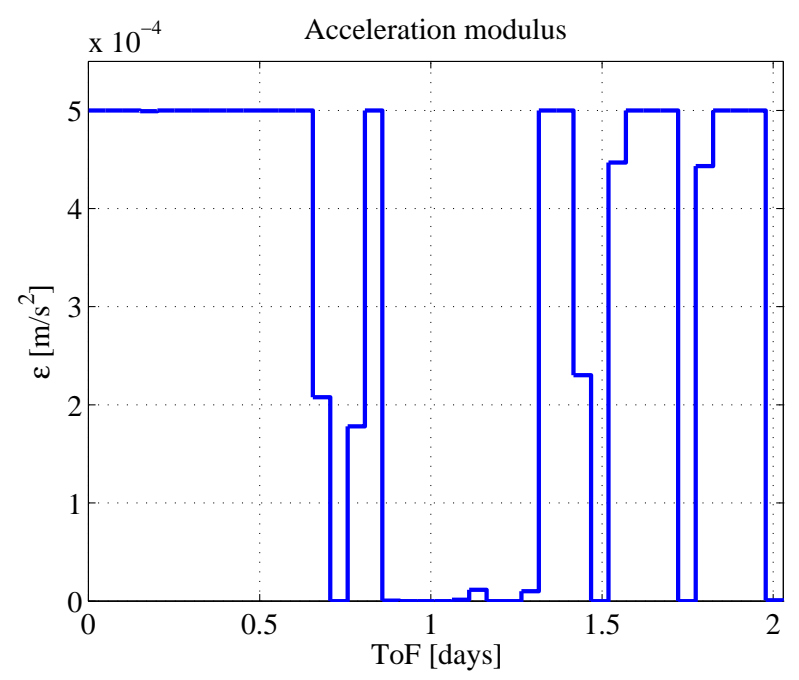

Fig. 19: Acceleration modulus $\varepsilon$ for LEO-ISS LT transfer. 


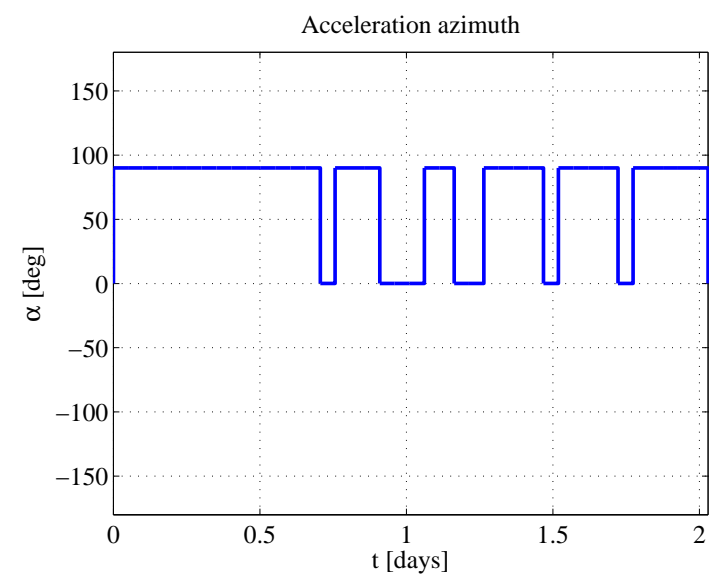

Fig. 20: Acceleration azimuth $\alpha$ for LEO-ISS LT transfer.

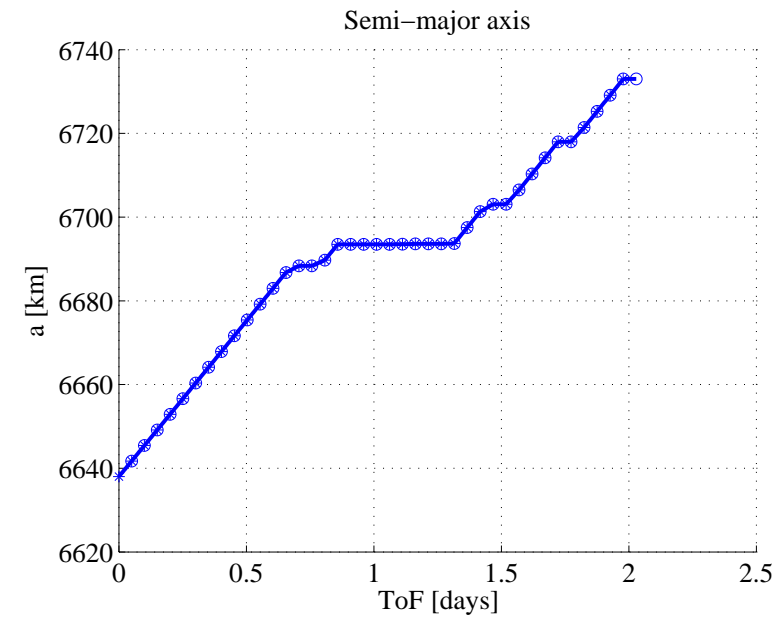

Fig. 21: Variation of Keplerian Elements for LEO-ISS LT transfer: $a$.

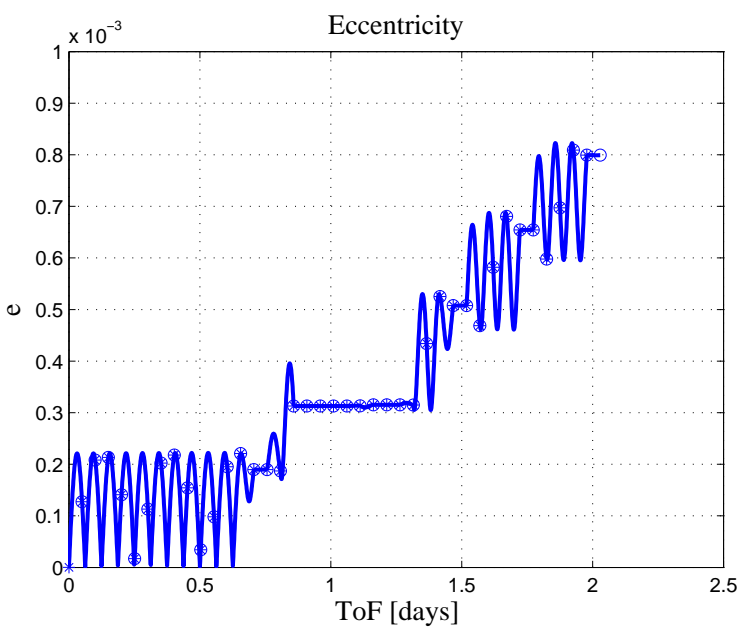

Fig. 22: Variation of Keplerian Elements for LEO-ISS LT transfer: $e$. 


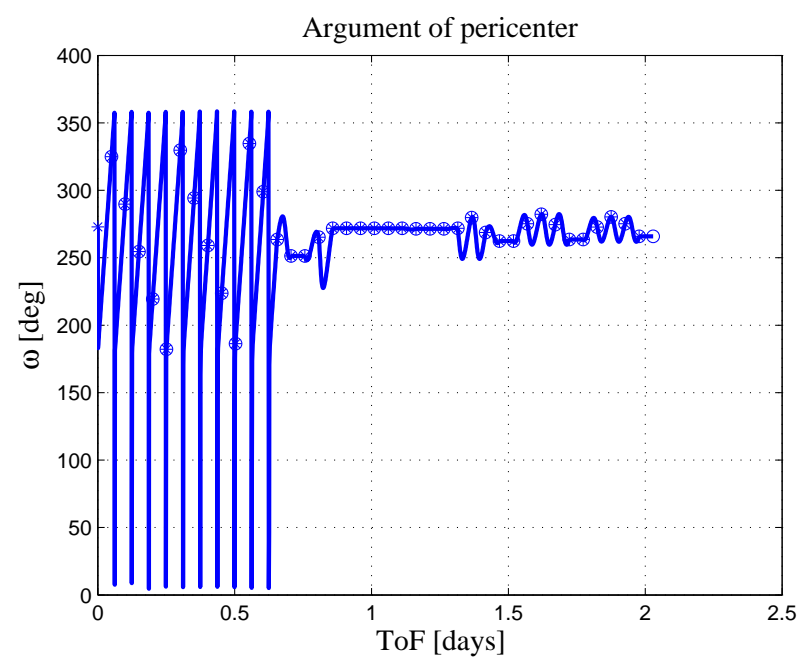

Fig. 23: Variation of Keplerian Elements for LEO-ISS LT transfer: $\omega$.

Fig. 19 and Fig. 20 show the time history of the acceleration modulus and azimuth respectively (the elevation plot has been omitted since it is constantly null). The acceleration azimuth (see Fig. 20), is almost constant at $90^{\circ}$, revealing a predominantly transversal and in-plane thrust component, while the thrust modulus in Fig. 20 shows a long thrusting arc along the initial part of the transfer which accounts for an almost linear increase of the semi-major axis as seen in Fig. 21. The eccentricity (see Fig. 22) shows a net increase during the transfer to reach the eccentricity of the arrival orbit, albeit it also has a periodic component during the thrusting spirals. The argument of pericenter (see Fig. 23) shows complete rotation of the orbit axis during the first long thrusting arc and small periodic oscillations in the other thrusting arcs.

\section{V.III Multiobjective Trajectory Optimisation}

Given its computational efficiency it is also possible to extend the field of application by using the proposed transcription method to solve a Global, Multi-Objective (MOO) Optimisation problem for trajectory design. As a first example, a simple direct, rendezvous transfer problem between Earth and Mars is considered. The aim is to find the transfers that are Pareto optimal with respect to the Time of Flight and the total $\Delta V$, within a certain range of departure dates and transfer times. The optimisation parameters in this case are simply the departure date $t_{0}$, the $T o F$ and the number of revolutions around the Sun. An agent-based multiobjective solver, called MACS ${ }^{28,29,30}$ (Multiagent Collaborative Search) was used to generate a number of Pareto optimal decision vectors. Then, for each decision vector, problem [12] is solved with fmincon. The boundary conditions for the solution of problem [12] are given by 
the ephemeris of the Earth at $t_{0}$ and those of Mars at $\left(t_{0}+T o F\right)$. The boundaries of the search space for the decision vector of the MOO problem are reported in Table 2.

\begin{tabular}{lcc}
\hline & Lower & Upper \\
\hline$t_{0}$ [MJD2000] & 5000 & 5779.94 \\
$T o F$ [days] & 100 & 1500 \\
$n_{\text {rev }}$ & 1 & 3 \\
\hline
\end{tabular}

Table 2: boundaries for optimization parameters for MO Earth-Mars transfer problem.

The number of revolutions is handled by MACS as a real variable and then is rounded to the nearest integer towards minus infinity when solving problem [12]. The trajectory was transcribed with 20 finite perturbative elements. The constraints violation and optimality tolerances for fmincon where slightly relaxed compared to the tests in the previous sections, with the former set to $10^{-6}$ and the latter to $10^{-4}$.

The MACS algorithm was run for 40000 function evaluations. The results of four different runs were combined to extract a good approximation of the Global Pareto Front. Fig. 24 and Fig. 25 report the solution points in the parameter space and the Pareto front respectively.

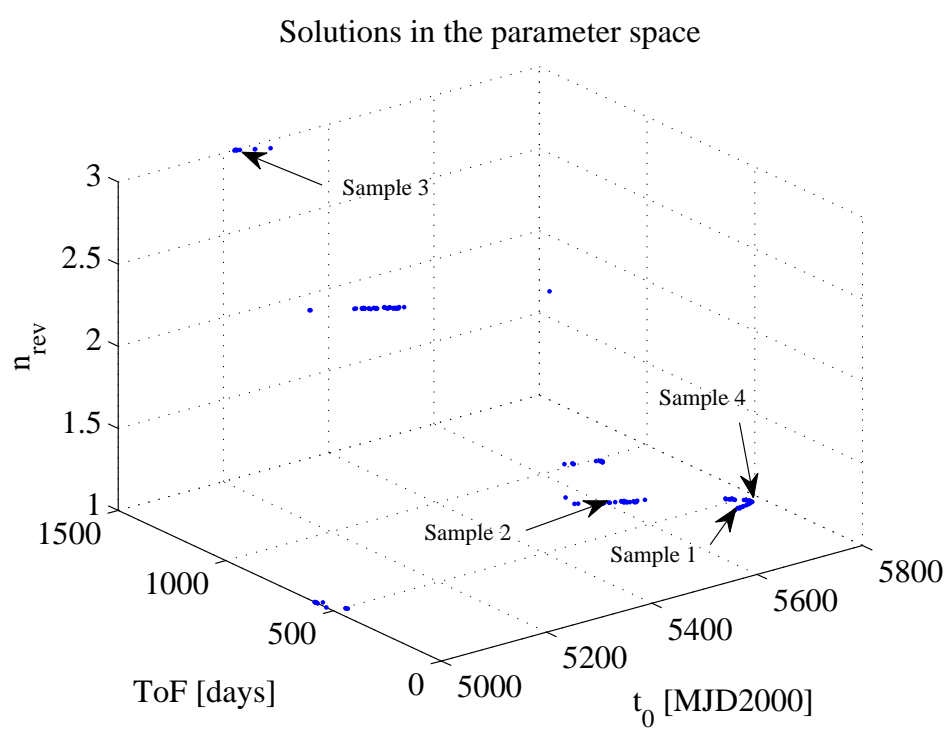

Fig. 24: Parameters of the solutions for MO Earth-Mars LT transfer problem. 


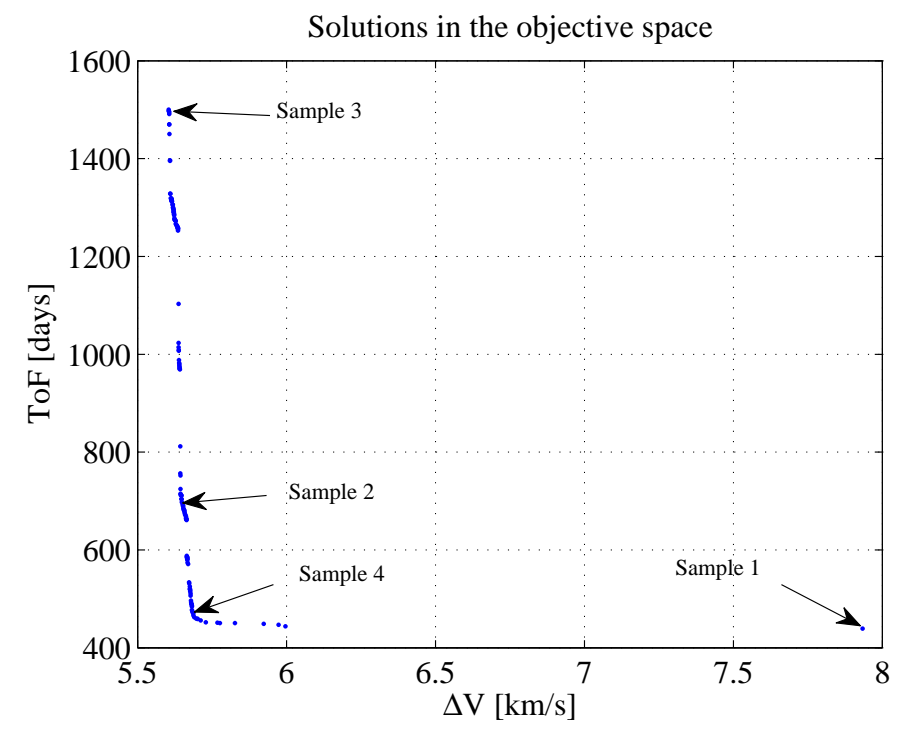

Fig. 25: Pareto front for MO Earth-Mars LT transfer problem.

The Pareto front presents some discontinuities, which are due to the discrete variable $n_{\text {rev }}$. In particular, the solutions with ToF up to 1000 days are all with only one complete revolution, while those with ToF between 1000 and 1400 days make two complete revolutions and finally the few over 1400 days make three complete revolutions. Fig. 26 to Fig. 33 show the trajectories and corresponding control profiles for three different sample solutions extracted from the Pareto set:

1. The solution with minimum $T o F$.

2. The solution in the central part of the Pareto Front.

3. The solution with minimum $\Delta V$.

4. The solution at the knee of the Pareto front: the solution is similar to 1 albeit with a lower $\Delta V$ and slightly higher $T o F$.

\begin{tabular}{lllll}
\hline Sample & 1 & 2 & 3 & 4 \\
\hline$t_{0}[$ MJD2000] & 5737.01 & 5606.82 & 5222.81 & 5779.94 \\
$t_{0}[$ UGT] & $16 / 09 / 2015$ & $09 / 05 / 2015$ & $20 / 04 / 2014$ & $29 / 10 / 2015$ \\
& $12: 16: 16.32$ & $7: 41: 13.92$ & $7: 33: 18.72$ & $03: 21: 36.00$ \\
ToF [days] & 439.21 & 704.25 & 1500 & 462.86
\end{tabular}




$\begin{array}{lllcr}n_{\text {rev }} & 1 & 1 & 3 & 1 \\ \Delta V[\mathrm{Km} / \mathrm{s}] & 7.9338 & 5.6474 & 5.6047 & 5.6902\end{array}$

Table 3: Summary of the four sample solutions

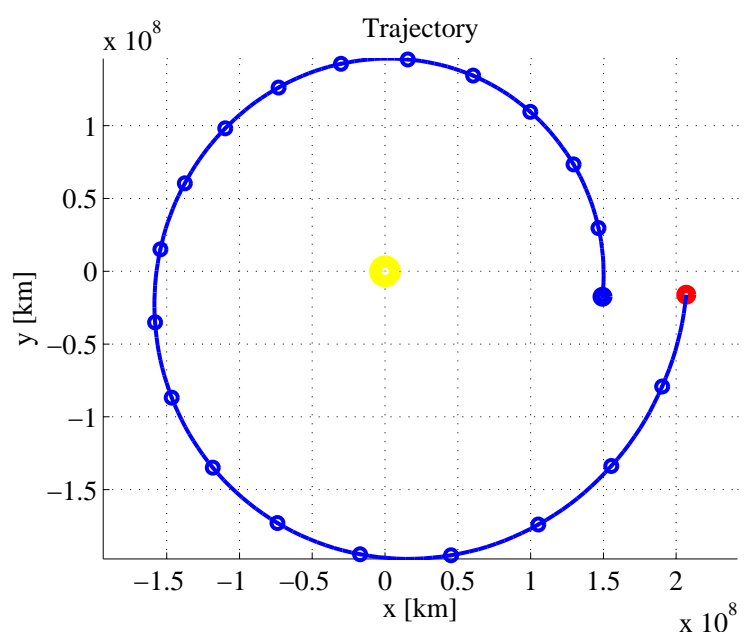

Fig. 26: Sample solution 1: trajectory.

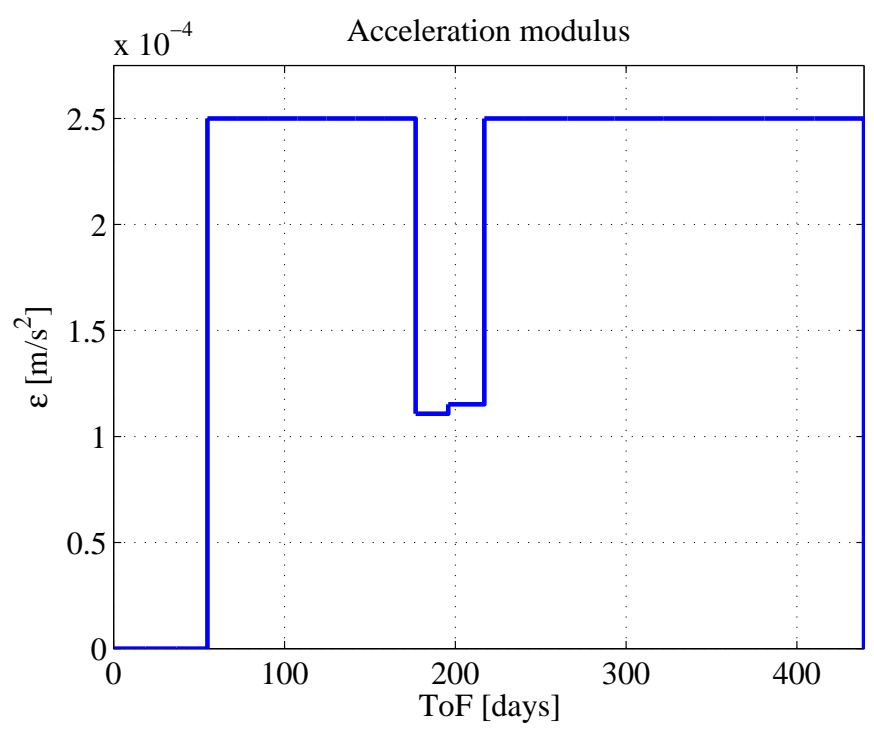

Fig. 27: Sample solution 1: acceleration modulus. 
The minimum ToF trajectory (see Fig. 26) reaches Mars in almost exactly one revolution. On the other hand, this requires continuous engine operation for almost the whole transfer, as shown in Fig. 27, which also translates into a $\Delta V$ cost of $7.93 \mathrm{~km} / \mathrm{s}$. Note that solutions with a lower $T o F$ could be possible, for $n_{\text {rev }}$ lower than 1 .

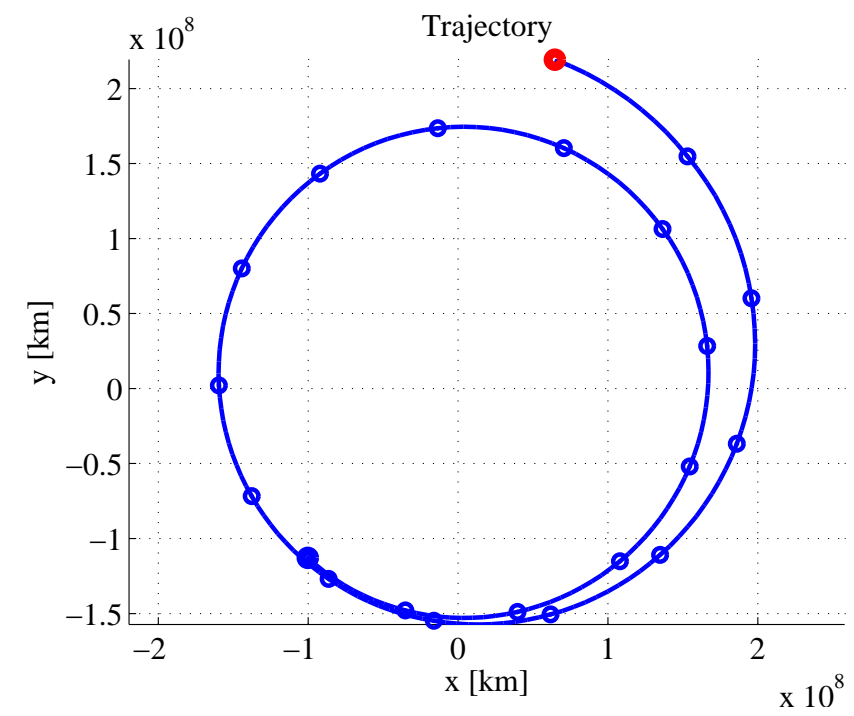

Fig. 28: Sample solution 2: trajectory.

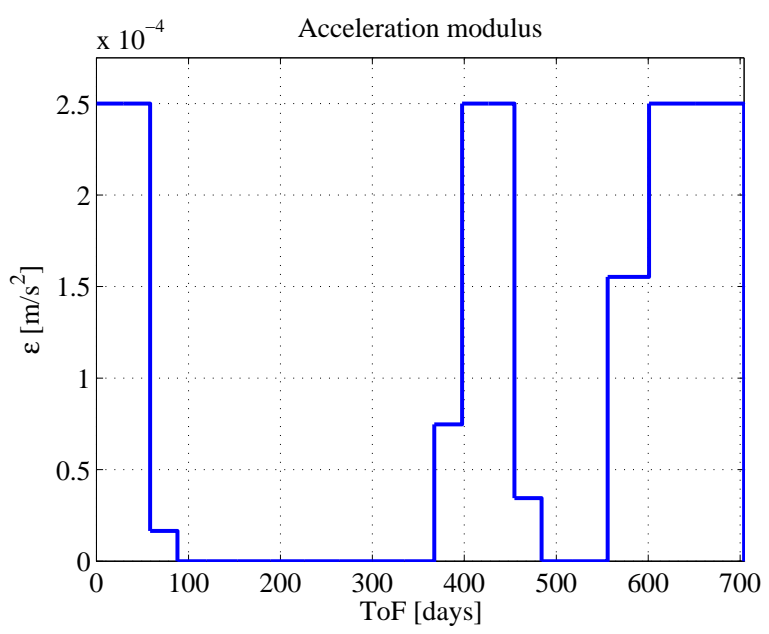

Fig. 29: Sample solution 2: acceleration modulus.

The second trajectory reaches Mars in 704 days with one revolution and a half and with three separate thrusting arcs (see Fig. 28 and Fig. 29). This allows for a better $\Delta V \operatorname{cost}$ of $5.65 \mathrm{~km} / \mathrm{s}$. 


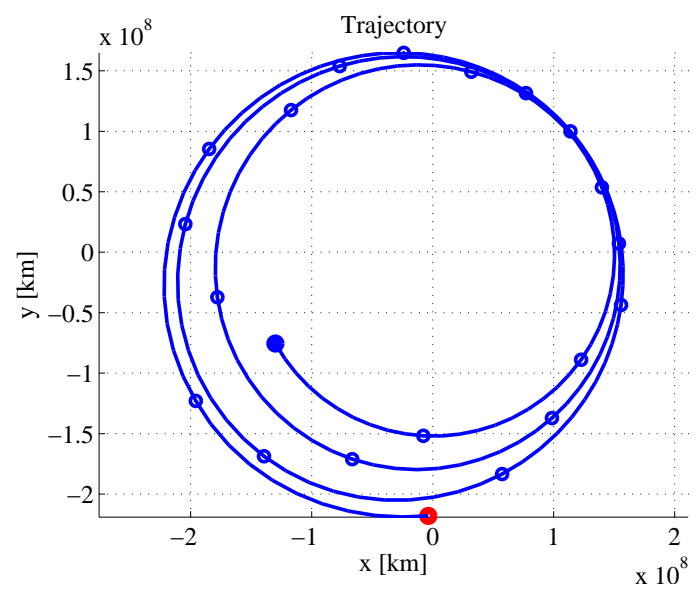

Fig. 30: Sample solution 3: trajectory.

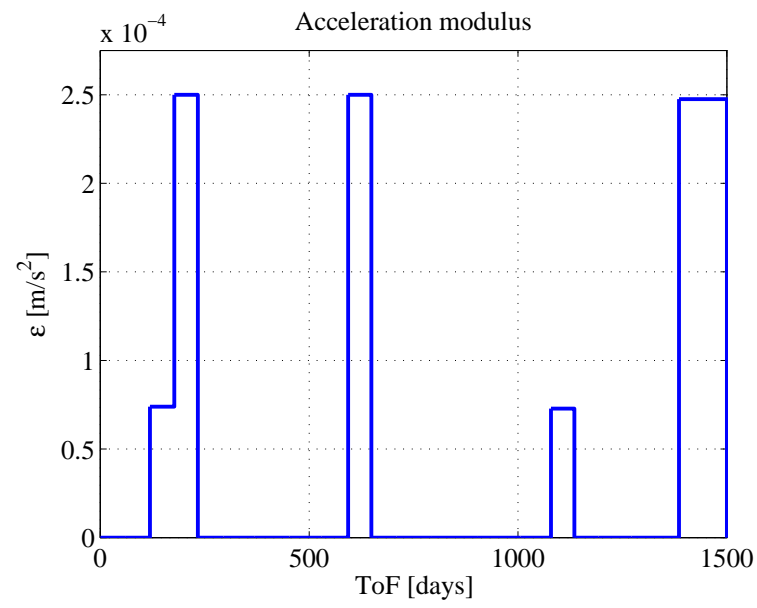

Fig. 31: Sample solution 3: acceleration modulus.

The minimum $\Delta V$ trajectory reaches Mars in 1500 days (the upper bound set for the $T o F$ ) with slightly more than three revolutions (see Fig. 30). As shown in Fig. 31, the four thrusting arcs are concentrated at periapsis and apoapsis, which allows for the mitigation of gravity losses and therefore a low $\Delta V \operatorname{cost}$ of $5.60 \mathrm{~km} / \mathrm{s}$. 


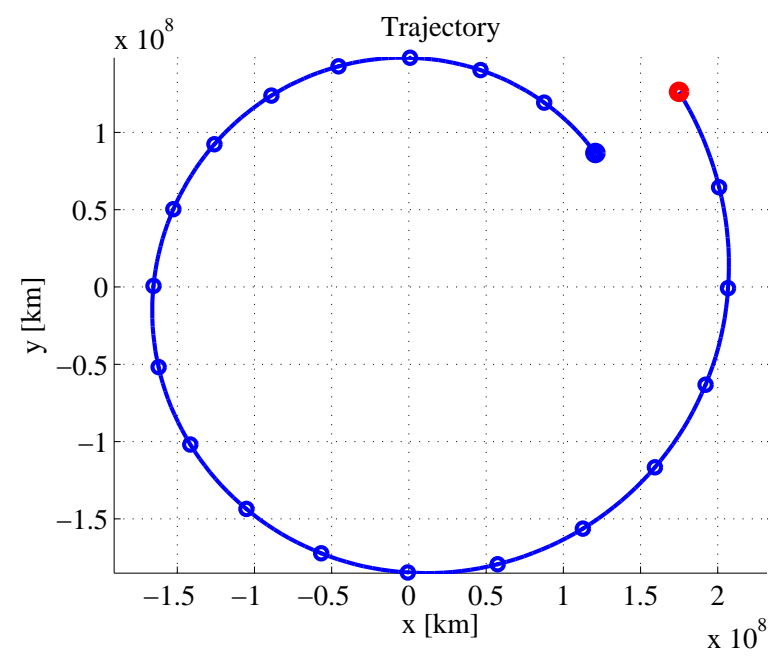

Fig. 32: Sample solution 4: trajectory.

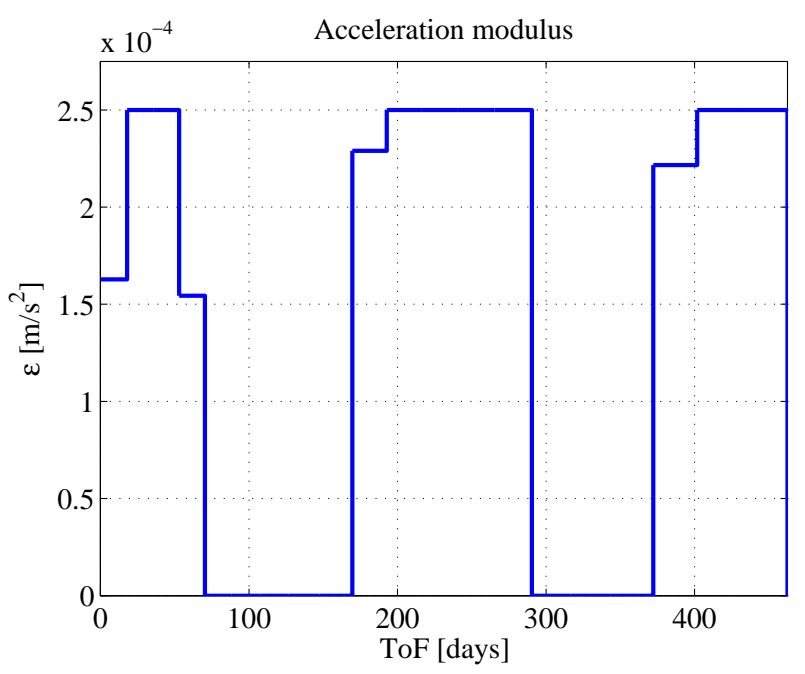

Fig. 33: Sample solution 4: acceleration modulus.

The knee solution has a similar phasing between the initial and final positions compared to the minimum ToF solution, see Fig. 32, but due to its higher ToF the total $\Delta V$ cost is lower. The thrust profile in Fig. 33 shows three separate thrusting arcs with a lower total thrust time compared to Fig. 27.

\section{CONCLUSION}

This work presented a novel numerical approach for Low Thrust trajectory transcription. The novel approach makes use of a first-order analytical solution of Gauss's planetary equations. The first order analytical solution was 
demonstrated to provide a fast and relatively accurate propagation of the perturbed Keplerian motion under the effect of a constant thrust. The first order approximation was implemented in a finite element formulation for the solution of two-point boundary value problems that was proven to be more computationally efficient and accurate than other state of the art methods. Furthermore, it was demonstrated how its computational efficiency makes the novel transcription method suitable for the solution of Global and Multi Objective Optimisation problems for LT trajectory design, in which the TPBVP needs to be solved thousands of times. Ongoing work is dedicated to the comparison of the DFPET transcription against other analytical solutions, such as the one derived from the Stark model ${ }^{20}$.

1 Betts, J.T, Survey of numerical methods for trajectory optimization, Journal of Guidance Control and Dynamics, Vol.21 No.2, 193-207, 1998.

2 Conway, B.A.: A brief survey of methods available for numerical optimization of spacecraft trajectories, 61th International Astronautical Congress of the International Astronautical Federation, Prague, Czech Republic, IAC-10-C1.2.1, 2010.

3 Azimov, D., Bishop, R.H.: Planetary capture using low-thrust propulsion, 16th International Symposium on Space Flight Dynamics. Pasadena, California, 2001.

4 Ranieri, C.L, Ocampo, C.A.: Optimization of roundtrip, time-constrained, finite burn trajectories via an indirect method, Journal of Guidance, Control and Dynamics, Vol.28 No.2, 306-314, 2005.

5 Casalino, L., Colasurdo, G. Pastrone, D.: Optimal Low-Thrust Escape Trajectories Using Gravity Assist, Journal of Guidance, Control, and Dynamics, Vol.22 No.5, 1999.

6 Betts, J.T., Erb, S.O.: Optimal Low-Thrust trajectories to the moon, SIAM Journal on Applied Dynamical Systems, Vol.2, 144, 2003.

7 Conway, B.A., Tang, S.: Optimization of low-thrust interplanetary trajectories using collocation and nonlinear programming, Journal of Guidance, Control, and Dynamics, Vol.18, No.3, 599-604, 1995.

8 Petropoulos, A.E., Longuski, J.M.: Shape-Based Algorithm for the Automated Design of Low-Thrust, Gravity-Assist Trajectories, Journal of Spacecraft and Rockets, Vol.41, No.5, 787-796, 2004.

9 Wall, B.J., Conway, B.A., Shape-based approach to low-thrust rendezvous trajectory design, Journal of Guidance, Control and Dynamics, Vol.32, No.1, 95-101, 2009. 
Vasile, M., De Pascale, P., Casotto, S.: On the optimality of a shape-based approach based on pseudoequinoctial elements, Acta Astronautica, Vol.61, No.1-6, 286-297, 2007.

11 Novak, D.M., Vasile, M.: Improved Shaping Approach to the Preliminary Design of Low-Thrust Trajectories, Journal of Guidance, Control and Dynamics, Vol.34, 128-147, 2011.

12 Sims, J.A., Flanagan, S. N.: Preliminary Design of Low-Thrust Interplanetary Missions, Paper AAS 99-338, 1999.

13 Hahn, G.D.: A modified Euler method for dynamic analyses, International Journal for Numerical Methods in Engineering, International Journal for Numerical Methods in Engineering, Vol.32, No.5, 943-955, 1991. Vavrina, M.A., Howell, K. C.: Global Low-Thrust Trajectory Optimization through Hybridization of a Genetic Algorithm and a Direct Method, AIAA/AAS Astrodynamics Specialist Conference pp. 18-21, 2008.

15 Yam, C.H., Di Lorenzo, D., Izzo, D.: Constrained Global Optimization of Low-Thrust Interplanetary Trajectories, Proceedings of the Twelfth conference on Congress on Evolutionary Computation (CEC 2010), 2010.

16

Sukhanov, A. A., Prado, A. F. B. de A.: Optimization of Transfers under Constraints on the Thrust Direction: II, Cosmic Research, Vol. 46, No. 1, 2008, pp. 51-60.

17 Battin, R.H.: An introduction to the mathematics and methods of astrodynamics, AIAA Education Series, 1987.

18 Kechichian, J.A.: Reformulation of Edelbaum's low-thrust transfer problem using optimal control theory, Journal of Guidance Control and Dynamics, Vol.20, 988-994, 1997.

19 Colombo, C., Vasile, M., Radice, G.: Semi-analytical solution for the optimal low-thrust deflection of NearEarth Objects, Journal of Guidance, Control and Dynamics, Vol.32, No.3, 796-809, 2009. Lantoine, G., Russell, R.P.: The Stark Model: An Exact, Closed-Form Approach to Low-Thrust Trajectory Optimization, $21^{\text {st }}$ International Symposium on Space Flight Dynamics, 2009.

21 Palmas, A.: Approximation of Low Thrust Trajectory Arcs by means of Perturbative Approaches, M.Sc. Thesis, Politecnico di Torino, July 2010.

22 Johnson, R.S., Singular Perturbation Theory, Springer, Boston, 2005.

23 Kevorkian J., Cole J.D.: Multiple Scale and Singular Perturbation Methods, Springer, New York, 1996. 

1999.

25 Vasile M. Bernelli-Zazzera F., Optimizing Low-Thrust and Gravity Assist Maneuvres to Design Interplanetary Trajectories, The Journal of the Astronautical Sciences. Vol. 51, No. 1, January-March 2003. Vasile M. Direct Transcription by FET for Optimal Space Trajectory Design. Internal Report DIA-SR 99-02, 1999.

27 Gill, P.E., Murray, W., Saunders, M.A.: SNOPT: An SQP algorithm for large-scale constrained optimization, SIAM Journal on Optimization, Vol.12 No.4, 979-1006, 2002.

28 Vasile M., A Hybrid Multi-Agent Collaborative Search Applied to the Solution of Space Mission Design Problems. Global Optimization Workshop, September 18-22, 2005, Almeria, Spain.

29 Vasile M., Hybrid Behavioural-Based Multiobjective Space Trajectory Optimization. on Multi-Objective Memetic Algorithms, Springer Series of Studies in Computational Intelligence, Series: Studies in Computational Intelligence, Vol. 171, Goh, Chi-Keong; Ong, Yew-Soon; Tan, Kay Chen (Eds.) 2009, XII, 404 p. 227 illus., Hardcover, ISBN: 978-3-540-88050-9.

30 Vasile, M., Zuiani, F.: A Hybrid Multiobjective Optimization Algorithm Applied to Space Trajectory Optimization, Proceedings of the Twelfth conference on Congress on Evolutionary Computation (CEC 2010), 2010. 